\title{
Hamiltonian Dynamics of Linearly Polarized Gowdy Models Coupled to Massless Scalar Fields
}

\author{
J. Fernando Barbero G.* and Daniel Gómez Verge日 \\ Instituto de Estructura de la Materia, \\ CSIC, Serrano 123, 28006 Madrid, Spain \\ Eduardo J. S. Villaseñon \\ Grupo de Modelización y Simulación Numérica, Universidad Carlos III de Madrid, \\ Avda. de la Universidad 30, 28911 Leganés, Spain and \\ Instituto de Estructura de la Materia, \\ CSIC, Serrano 123, 28006 Madrid, Spain
}

(Dated: July 23, 2007)

\begin{abstract}
The purpose of this paper is to analyze in detail the Hamiltonian formulation for the compact Gowdy models coupled to massless scalar fields as a necessary first step towards their quantization. We will pay special attention to the coupling of matter and those features that arise for the $\mathbb{S}^{1} \times \mathbb{S}^{2}$ and $\mathbb{S}^{3}$ topologies that are not present in the well studied $\mathbb{T}^{3}$ case -in particular the polar constraints that come from the regularity conditions on the metric. As a byproduct of our analysis we will get an alternative understanding, within the Hamiltonian framework, of the appearance of initial and final singularities for these models.
\end{abstract}

PACS numbers: 04.20.Fy, 04.60.Kz

\section{INTRODUCTION}

Symmetry reductions are a way to gain useful insights for difficult problems in classical and quantum general relativity. In this respect the two Killing vector reductions provided by the so called Gowdy models are specially attractive because they have a cosmological interpretation and share some interesting features with similar reductions such as the EinsteinRosen waves -in particular their solvability- both at the classical and quantum regimes. Most of the work on these models, after the initial papers by Gowdy [1, 2], has profusely analyzed those corresponding to the 3-torus spatial topology and, in fact, this is by far the preferred choice to discuss quantization issues [3, 4, 15, 6, 7, [8, 9, 10, 11, 12, 13, 14, 15]. The other possible closed (compact and without boundary) topologies, the three-handle $\mathbb{S}^{1} \times \mathbb{S}^{2}$, the three-sphere $\mathbb{S}^{3}$, and the lens spaces $L(p, q)$, are interesting in their own right. From the physical point of view their most salient feature is the fact that they describe cosmological models with both initial and final singularities. For this reason they will become useful test

*fbarbero@iem.cfmac.csic.es dgvergel@iem.cfmac.csic.es 抽anche@math.uc3m.es 
beds for issues related to quantization in cyclic universes.

The first step towards quantization is the Hamiltonian formulation of the model at hand. This is specially so for constrained systems where the identification of the relevant constraints is a necessary first step either to attempt a phase space reduction, a gauge fixing, or a quantization à la Dirac where the physical Hilbert space is identified as the kernel of suitable self-adjoint operators representing the constraints.

Our goal in this paper is to perform a detailed Hamiltonian analysis of the compact Gowdy models coupled to massless scalar fields, extending in several ways previous results on this subject [16, 17]. Adding matter fields to the system is a way to enrich these models and get closer to physically realistic situations. Here we will work in the spirit of previous treatments for other two Killing vector reductions [18, 19], paying close attention to the constraint analysis, gauge fixing, and deparameterization. To our knowledge the Hamiltonian analysis, for the vacuum $\mathbb{S}^{1} \times \mathbb{S}^{2}$ and $\mathbb{S}^{3}$ Gowdy models, has only been addressed in a partial way in [20] where the authors give Hamiltonians for these systems. However they do not provide the detailed phase space description (constraints, gauge fixing, and so on) necessary to understand relevant geometrical issues. Also their reduced phase space treatment does not allow to follow other roads to quantization such as Dirac's approach or the viewpoint pioneered by Varadarajan in [21]. Among several issues we want to find out how the topology of the spatial slices affects the definition of the constraints, and how the coupling of massless scalar fields is realized in the different topologies. Along the way we also want to understand in a detailed way, and within the Hamiltonian setting, the mechanisms leading to the appearance of final singularities. Our results can be immediately particularized to the vacuum situation.

The paper is structured as follows. After this introduction we will review in section [I the main points concerning the Geroch reduction for polarized Gowdy models coupled to massless scalar matter fields. In particular we will show that this reduction, and a subsequent conformal transformation, allows us to interpret these models as $2+1$ gravity coupled to a set of massless scalar fields with axial symmetry. Some details related to this reduction vary depending on the topologies (in particular those related to the quotient spatial manifold) and will be commented separately for each case.

Section III will be devoted to discuss the Gowdy $\mathbb{T}^{3}$ models coupled to massless scalar fields extending the previous treatments for the vacuum case [9, 14] (and similar models such as Einstein-Rosen waves [16, 17]). Along the way we will clarify some issues related to deparameterization and the appearance of singularities in the $3+1$ dimensional metrics. This section will be the basis of the treatment that we will follow to study the other possible topologies.

Section [V] will be devoted to the Hamiltonian formulation of Gowdy models in $\mathbb{S}^{1} \times \mathbb{S}^{2}$ coupled to massless scalars. Here we will have to pay special attention to the identification of the regularity conditions that the basic fields describing the model must satisfy as a consequence of the regularity conditions on the metric. As we will see the constraints that are relevant here are different from the ones present for the 3-torus due to the presence of a symmetry axis in the spatial manifold. In particular we will get what we will refer to as "polar constraints" involving the values of the basic fields at the poles of the two dimensional sphere that appears as the quotient space after performing a Geroch reduction. As we will show they are first class and play a relevant role to guarantee the differentiability of the other constraints. Another issue that will be discussed is how the deparameterization achieved by a partial gauge fixing works for this model and how one can arrive at a reduced phase 
space description. We will see that, as it also happens in the $\mathbb{T}^{3}$ case, the dynamics of the system is described by a time dependent Hamiltonian though the time dependence now is different and reflects the appearance of initial and final singularities. In fact, as a result of our analysis, we will get a geometric understanding of this fact in terms of the geometry of the constraint hypersurface in phase space.

After this we will perform a similar analysis in section $\nabla$ for the $\mathbb{S}^{3}$ topology. Here the main difference stems from the fact that we will be forced to perform the Geroch reduction needed to describe the model in $2+1$ dimensions by using a Killing field whose norm vanishes on a circle $\mathbb{S}^{1}$. This will introduce some modifications in our description and will change the analysis of the relevant regularity conditions for the metric. Nevertheless we will find out that the final description is quite similar to the one corresponding to the three handle discussed above.

The detailed quantization of the $\mathbb{S}^{1} \times \mathbb{S}^{2}$ and $\mathbb{S}^{3}$ Gowdy models will be carried out elsewhere. A fact that will play a relevant role there is the possibility of describing the compact Gowdy models in the different topologies as field theories in certain conformally stationary curved backgrounds. As this point of view is also useful to understand some of the issues discussed in the paper from a different perspective we will show in section VI how this can be done.

We end the paper in section VII with a discussion of the main results and suggestions for future work on this subject.

\section{GENERAL FEATURES OF COMPACT GOWDY MODELS: GEROCH REDUCTION AND $2+1$ DIMENSIONAL FORMULATION}

Let us consider a smooth, effective, and proper action of the biparametric Lie Group $G^{(2)}:=U(1) \times U(1)=\left\{\left(g_{1}, g_{2}\right)=\left(e^{i x_{1}}, e^{i x_{2}}\right) \mid x_{1}, x_{2} \in \mathbb{R}(\bmod 2 \pi)\right\}$ on a compact, connected, and oriented 3-manifold ${ }^{(3)} \Sigma$. It can be shown [22, 23] that this action is unique up to automorphisms of $G^{(2)}$ and diffeomorphisms of ${ }^{(3)} \Sigma$. The spatial manifold ${ }^{(3)} \Sigma$ is then restricted to have the topology of a three-torus $\mathbb{T}^{3}$, a three-handle $\mathbb{S}^{1} \times \mathbb{S}^{2}$, the three-sphere $\mathbb{S}^{3}$, or the lens spaces $L(p, q)$ (that can be studied by imposing discrete symmetries on the $\mathbb{S}^{3}$ case).

Let us take a four manifold ${ }^{(4)} \mathcal{M}$ diffeomorphic to $\mathbb{R} \times{ }^{(3)} \Sigma$ and such that $\left({ }^{(4)} \mathcal{M},{ }^{(4)} g_{a b}\right)$ is a globally hyperbolic spacetime endowed with a Lorentzian metric ${ }^{1}{ }^{(4)} g_{a b}$. Let us further require that $G^{(2)}$ acts by isometries on the spatial slices of ${ }^{(4)} \mathcal{M}$. In this paper we will focus on the so called linearly polarized case, hence, the isometry group will be generated by a pair of mutually orthogonal, commuting, spacelike, and globally defined hypersurface-orthogonal Killing vector fields $\left(\xi^{a}, \sigma^{a}\right)$.

Let us consider now the Einstein-Klein-Gordon equations

$$
{ }^{(4)} R_{a b}=8 \pi G_{N}(\mathrm{~d} \phi)_{a}(\mathrm{~d} \phi)_{b}, \quad{ }^{(4)} g^{a b(4)} \nabla_{a}{ }^{(4)} \nabla_{b} \phi=0
$$

corresponding to (3+1)-dimensional gravity minimally coupled to a zero rest mass scalar field

\footnotetext{
${ }^{1}$ Throughout the paper we will use the Penrose abstract index convention with tangent space indices belonging to the beginning of the Latin alphabet 24]. Lorentzian spacetime metrics will have signature $(-+++)$ and the conventions for the curvature tensors are those of Wald [25].
} 
$\phi$ symmetric under the diffeomorphisms generated by the Killing fields $\left(\mathcal{L}_{\xi} \phi=\mathcal{L}_{\sigma} \phi=0\right.$, $\left.\mathcal{L}_{\xi}{ }^{(4)} g_{a b}=\mathcal{L}_{\sigma}{ }^{(4)} g_{a b}=0\right)$. Here ${ }^{(4)} R_{a b}$ and ${ }^{(4)} \nabla_{a}$ denote the Ricci tensor and the metric connection associated to ${ }^{(4)} g_{a b}$, respectively. The exterior derivative of the scalar field $\phi$ is denoted by $(\mathrm{d} \phi)_{a}$ and $G_{N}$ is the Newton constant.

In order to get a simplified, lower dimensional description we will perform a Geroch reduction [26] by taking advantage of the existence of Killing vector fields. The possibility of finding the necessary non-vanishing Killing field $\xi^{a}$ will depend, as we will see later, on the spatial topology that we consider. In some cases the appropriate Killing vectors vanish on 2dimensional submanifolds but, nevertheless, we will be able to use Geroch's procedure even in this situation. The idea is to find a suitable reduction on the manifold ${ }^{(3)} \mathcal{M}={ }^{(4)} \tilde{\mathcal{M}} / U(1)$, diffeomorphic to $\mathbb{R} \times{ }^{(2)} \Sigma$, where ${ }^{(4)} \tilde{\mathcal{M}}$ denotes the set of points in ${ }^{(4)} \mathcal{M}$ in which $\xi^{a}$ is nonvanishing, and reintroduce the removed points (the symmetry axis) as a boundary where the fields must satisfy certain regularity conditions. In the present situation hypersurface orthogonality will allow us to view ${ }^{(3)} \mathcal{M}$ as an embedded submanifold, everywhere orthogonal to the closed orbits of $\xi^{a}$, and endowed with the induced metric ${ }^{(3)} g_{a b}:={ }^{(4)} g_{a b}-\lambda_{\xi}^{-1} \xi_{a} \xi_{b}$, where $\lambda_{\xi}:={ }^{(4)} g_{a b} \xi^{a} \xi^{b}:=\xi_{a} \xi^{a}>0$.

In the linearly polarized case the twist of the Killing fields vanishes and the field equations can be written as those corresponding to a set of massless scalar fields coupled to $(2+1)$ gravity by performing the conformal transformation $g_{a b}:=\lambda_{\xi}{ }^{(3)} g_{a b}$. The system (2.1) is then equivalent to

$$
R_{a b}=\frac{1}{2} \sum_{i}\left(\mathrm{~d} \phi_{i}\right)_{a}\left(\mathrm{~d} \phi_{i}\right)_{b}, \quad g^{a b} \nabla_{a} \nabla_{b} \phi_{i}=0, \quad \mathcal{L}_{\sigma} g_{a b}=0, \quad \mathcal{L}_{\sigma} \phi_{i}=0,
$$

where $R_{a b}$ and $\nabla_{a}$ denote, respectively, the Ricci tensor and the Levi-Civita connection associated to $g_{a b}$ (all of them three dimensional objects), we have defined ${ }^{2} \phi_{1}:=\log \lambda_{\xi}$, $\phi_{2}:=\sqrt{16 \pi G_{N}} \phi$, and we must remember that we have the additional symmetry generated by the remaining Killing vector field $\sigma^{a}$. Notice that (2.2) are formally symmetric under the exchange of the gravitational and matter scalars. However, it is important to realize that for some of the topologies that we will discuss, these fields may be subject to different regularity conditions in the gravitational and matter sectors that effectively break the symmetry among them. The relevant details for each spatial topology will be given in the corresponding section.

In order to obtain the Hamiltonian formulations for the models that we are considering here we want to derive the previous equations from an action principle. To this end we introduce the $(2+1)$-dimensional Einstein-Hilbert action corresponding to gravity coupled

\footnotetext{
$22+1$ massless scalar fields will be denoted be the subindex $i=1,2$. The subindex $i=1$ will label the gravitational scalar that encodes the local gravitational degrees of freedom in Gowdy models and the subindex $i=2$ will label the original $3+1$ matter scalar. It is completely straightforward to couple any number $N$ of massless scalar fields, in practice this can be done by supposing that the index $i$ runs from 1 to $N$.
} 
to massless scalars

$$
\begin{aligned}
{ }^{(3)} S\left(g_{a b}, \phi_{i}\right)= & \frac{1}{16 \pi G_{3}} \int_{\left(t_{0}, t_{1}\right) \times()_{\Sigma}}{ }^{(3)} \mathrm{e}|g|^{1 / 2}\left(R-\frac{1}{2} \sum_{i} g^{a b}\left(\mathrm{~d} \phi_{i}\right)_{a}\left(\mathrm{~d} \phi_{i}\right)_{b}\right) \\
& +\frac{1}{8 \pi G_{3}} \int_{\left\{t_{0}\right\} \times(2)}{ }_{\Sigma \cup\left\{t_{1}\right\} \times\left({ }^{(2)} \Sigma\right.}{ }^{(2)} \mathrm{e}|h|^{1 / 2} K .
\end{aligned}
$$

Here $R$ denotes the Ricci scalar associated to $g_{a b} . K$ and $h_{a b}$ are, respectively, the trace of the second fundamental form $K_{a b}$ (defined by the exterior normal unit vector $n^{a}$ ), and the induced 2-metric on the boundary $\left\{t_{0}\right\} \times{ }^{(3)} \Sigma \cup\left\{t_{1}\right\} \times{ }^{(3)} \Sigma$. Finally $G_{3}$ denotes the Newton constant per unit length in the direction of the $\xi$-symmetric orbits. We have restricted the integration region to an interval $\left[t_{0}, t_{1}\right]$, where $t$ defines a global coordinate on $\mathbb{R}$. The action is written with the help of a fiducial (i.e. non dynamical) volume form ${ }^{(3)} \mathrm{e}$ compatible with the canonical volume form ${ }^{(3)} \epsilon$ defined by the metric $g_{a b}$. This is given by ${ }^{3}{ }^{(3)} \epsilon=\left.\sqrt{|g|}\right|^{(3)} \mathrm{e}$. The volume form ${ }^{(3)} \epsilon$ induces a 2-form ${ }^{(2)} \epsilon_{a b}={ }^{(3)} \epsilon_{a b c} n^{c}$ on each slice $\{t\} \times{ }^{(2)} \Sigma$ that agrees with the volume associated to the 2-metric $h_{a b}$. We have also introduced a fixed volume 2 -form ${ }^{(2)} \mathrm{e}$ on $\{t\} \times{ }^{(2)} \sum$ such that ${ }^{(2)} \epsilon=\sqrt{|h|}{ }^{(2)} \mathrm{e}$, and verifies $\sqrt{|g|}^{(3)} \mathrm{e}_{a b c} n^{c}=\sqrt{|h|}{ }^{(2)} \mathrm{e}_{a b}$. We require that ${ }^{\left({ }^{3)}\right.} \mathrm{e}$ and ${ }^{(2)} \mathrm{e}$ be time-independent, i.e. $\mathcal{L}_{t}{ }^{(3)} \mathrm{e}=0, \mathcal{L}_{t}{ }^{(2)} \mathrm{e}=0$ where $\mathcal{L}_{t}$ denotes Lie derivative along $t^{a}:=(\partial / \partial t)^{a}$. We also demand them to be invariant under the action of the remaining Killing vector field. In particular, given the $(2+1)$-dimensional splitting of ${ }^{(3)} \Sigma$ it is natural to choose ${ }^{(3)} \mathrm{e}=\mathrm{d} t \wedge{ }^{(2)} \mathrm{e}$, with $\mathcal{L}_{t}{ }^{(2)} \mathrm{e}=\mathcal{L}_{\sigma}{ }^{(2)} \mathrm{e}=0$ [25].

For all the different topologies, using the Stokes theorem, we get

$$
{ }^{(3)} S\left(g_{a b}, \phi_{i}\right)=\frac{1}{16 \pi G_{3}} \int_{t_{0}}^{t_{1}} \mathrm{~d} t \int_{(2)_{\Sigma}}{ }_{(2)} \mathrm{e}|g|^{1 / 2}\left({ }^{(2)} R+K_{a b} K^{a b}-K^{2}-\frac{1}{2} \sum_{i} g^{a b}\left(\mathrm{~d} \phi_{i}\right)_{a}\left(\mathrm{~d} \phi_{i}\right)_{b}\right)
$$

where we have used the relation $R={ }^{(2)} R+K_{a b} K^{a b}-K^{2}+2 \nabla_{a}\left(n^{a} K-n^{b} \nabla_{b} n^{a}\right)$ and ${ }^{(2)} R$ denotes the Ricci scalar associated to $h_{a b}$. Our strategy in the different topologies that we will study in the paper will be to write down an action of this type, adapted to the peculiarities of the different spatial topologies (in particular those originating in the different sets of regularity conditions that we will have to consider) and use it to derive a Hamiltonian formulation for the system.

\section{III. $\quad \mathbb{T}^{3}$ GOWDY MODELS COUPLED TO MASSLESS SCALARS}

The $\mathbb{T}^{3}$ Gowdy model is, by far, the most studied to date both at the classical and quantum levels [1, 2, 3, $4,45,6,7,6,19,10,11,12,13,14,15,23]$. We will consider in this section the coupling of some types of matter fields and the most important aspects of its Hamiltonian treatment paying special attention to the deparameterization and reduced Hamiltonian description.

Let us start by considering the orientable 3-manifold ${ }^{(3)} \Sigma=\mathbb{T}^{3}=\mathbb{S}^{1} \times \mathbb{S}^{1} \times \mathbb{S}^{1}$, whose

\footnotetext{
${ }^{3}$ In any basis where the nonvanishing components of ${ }^{(3)} \epsilon$ have the values $\pm 1,|g|^{1 / 2}$ is equivalent to the square of the absolute value of the determinant of the matrix of the metric in that basis.
} 
points we parameterize in the form $\left(z_{1}, z_{2}, z_{3}\right)=\left(e^{i \theta}, e^{i \sigma}, e^{i \xi}\right)$ with $\theta, \sigma, \xi \in \mathbb{R}(\bmod 2 \pi)$. In particular, we endow ${ }^{(3)} \Sigma$ with the standard volume form $\mathrm{d} \theta \wedge \mathrm{d} \sigma \wedge \mathrm{d} \xi$. We define the following (left) $G^{(2)}$-group action

$$
\left(g_{1}, g_{2}\right) \cdot\left(z_{1}, z_{2}, z_{3}\right)=\left(e^{i x_{1}}, e^{i x_{2}}\right) \cdot\left(e^{i \theta}, e^{i \sigma}, e^{i \xi}\right):=\left(e^{i \theta}, e^{i\left(x_{1}+\sigma\right)}, e^{i\left(x_{2}+\xi\right)}\right) .
$$

We can consider now the group orbits defined by the commuting subgroups $\left(g_{1}, g_{2}\right)=\left(e^{i x}, 1\right)$, $\left(g_{1}, g_{2}\right)=\left(1, e^{i x}\right), x \in \mathbb{R}(\bmod 2 \pi)$

$$
\begin{aligned}
& \left(e^{i x}, 1\right) \cdot\left(e^{i \theta}, e^{i \sigma}, e^{i \xi}\right)=\left(e^{i \theta}, e^{i(x+\sigma)}, e^{i \xi}\right) \\
& \left(1, e^{i x}\right) \cdot\left(e^{i \theta}, e^{i \sigma}, e^{i \xi}\right)=\left(e^{i \theta}, e^{i \sigma}, e^{i(x+\xi)}\right)
\end{aligned}
$$

and their corresponding tangent vectors at each point of $\mathbb{T}^{3}$ obtained by differentiating the previous expressions with respect to $x$ at $x=0$

$$
\begin{aligned}
& \left(0, i e^{i \sigma}, 0\right), \\
& \left(0,0, i e^{i \xi}\right) .
\end{aligned}
$$

Let us consider the four manifold ${ }^{(4)} \mathcal{M} \simeq \mathbb{R} \times \mathbb{T}^{3}$. We introduce now three smooth vector fields $\theta^{a}, \sigma^{a}$ and $\xi^{a}$, tangent to the embedded submanifolds $\{t\} \times \mathbb{T}^{3}$ (here $t$ is a global coordinate on $\mathbb{R})$. In order to do this, let us fix $t_{0} \in \mathbb{R}$ and define on $\left\{t_{0}\right\} \times \mathbb{T}^{3}$ the 3dimensional vector fields ${ }^{4}(\partial / \partial \theta)^{a},(\partial / \partial \sigma)^{a}$, and $(\partial / \partial \xi)^{a}$ given in the description of ${ }^{(3)} \Sigma$ at the beginning of this section. We extend them to ${ }^{(4)} \mathcal{M}$ by Lie dragging along a smooth vector field $t^{a}$ defined ${ }^{5}$ as the tangent vector to a smooth congruence of curves transverse to the slices $\{t\} \times \mathbb{T}^{3}$. Notice that given a one parameter family of diffeomorphisms $f_{t}$ we have $f_{t *}[\xi, \sigma]^{a}=\left[f_{t *} \xi, f_{t *} \sigma\right]^{a}$ so we guarantee that the extended fields commute everywhere. The 4tuple $\left(t^{a}, \theta^{a}, \sigma^{a}, \xi^{a}\right)$ defines then a paralelization of ${ }^{(4)} \mathcal{M}$. Here $\theta^{a}$ is the vector field obtained by extending $(\partial / \partial \theta)^{a}$ to the four-dimensional manifold ${ }^{(4)} \mathcal{M} ; \sigma^{a}$ and $\xi^{a}$ are obtained by the same procedure. Once we have introduced these vector fields on ${ }^{(4)} \mathcal{M}$ as background objects we restrict ourselves to working with metrics ${ }^{(4)} g_{a b}$ satisfying the following conditions:

1. The action of the group $G^{(2)}$ on ${ }^{(4)} \mathcal{M}$ defined by $\left(g_{1}, g_{2}\right) \cdot(t, p)=\left(t,\left(g_{1}, g_{2}\right) \cdot p\right), t \in \mathbb{R}$, $p \in \mathbb{T}^{3}$, with $\left(g_{1}, g_{2}\right) \cdot p$ defined above, is an action by isometries, i.e. $\xi^{a}$ and $\sigma^{a}$ are Killing vector fields $\left(\mathcal{L}_{\xi}^{(4)} g_{a b}=0, \mathcal{L}_{\sigma}^{(4)} g_{a b}=0\right)$.

2. $t$ is a global time function, i.e. ${ }^{(4)} g^{a b}(\mathrm{~d} t)_{b}$ is a timelike vector field. From now on we will consider the manifold ${ }^{(4)} \mathcal{M}$ to be endowed with a time orientation such that this vector field is past-directed.

3. $\{t\} \times \mathbb{T}^{3}$ are spacelike hypersurfaces for all $t \in \mathbb{R}$. In particular $\lambda_{\xi}:={ }^{(4)} g_{a b} \xi^{a} \xi^{b}>0$, $\lambda_{\sigma}:={ }^{(4)} g_{a b} \sigma^{a} \sigma^{b}>0$.

4. $\xi^{a}$ and $\sigma^{a}$ are hypersurface orthogonal (this defines the so called linearly polarized case). This condition means that the twist of the two fields vanishes. This will

\footnotetext{
${ }^{4}$ Notice that even though we use a coordinate notation these are globally defined vector fields on the spatial manifolds $\left\{t_{0}\right\} \times \mathbb{T}^{3}$.

${ }^{5}$ In particular, take $t^{a}:=(\partial / \partial t)^{a}$.
} 
ultimately allow us to simplify the field equations and describe the system as a simple theory of scalar fields.

Two simple but important results that can be proved at this point as a consequence of the first are the following:

i) If $\xi^{a}$ and $\sigma^{a}$ are Killing vectors and $[\xi, \sigma]^{a}=0$ then $\mathcal{L}_{\sigma}\left({ }^{(4)} g_{a b}-\xi_{a} \xi_{b} / \lambda_{\xi}\right)=0$;

ii) Furthermore, if we define the vector $X^{a}$ orthogonal to $\xi^{a}$ as $X^{a}:=\sigma^{a}-\xi^{a}\left(\xi^{b} \sigma_{b}\right) / \lambda_{\xi}$ it satisfies $[\xi, X]^{a}=0$ and also $\mathcal{L}_{X}\left({ }^{(4)} g_{a b}-\xi_{a} \xi_{b} / \lambda_{\xi}\right)=0$. This means that, without loss of generality, we can work with everywhere orthogonal and commuting Killing vector fields $\xi^{a}$ and $\sigma^{a}$. In fact, we impose

5. $\left(\theta^{a}, \sigma^{a}, \xi^{a}\right)$ are mutually ${ }^{(4)} g$-orthogonal vector fields.

After we perform the Geroch reduction with respect to the field $\xi^{a}$ as described above we end up with a set of equations that can be obtained from a $2+1$ dimensional action of the type (2.4) with ${ }^{(2)} \Sigma=\mathbb{T}^{2}=\mathbb{S}^{1} \times \mathbb{S}^{1}$. Since the remaining Killing vector field $\sigma^{a}$ is still hypersurface orthogonal, and non-vanishing, the corresponding space of orbits ${ }^{(2)} \mathcal{M}:=$ ${ }^{(3)} \mathcal{M} / U(1) \simeq \mathbb{R} \times \mathbb{S}^{1}$ can be identified as an embedded hypersurface in ${ }^{(3)} \mathcal{M}$ everywhere orthogonal to the (closed) orbits of $\sigma^{a}$. The induced 2-metric of signature $(-+)$ on ${ }^{(2)} \mathcal{M}$ can be written

$$
s_{a b}=g_{a b}-\tau^{-2} \sigma_{a} \sigma_{b},
$$

where $\tau^{2}:=g_{a b} \sigma^{a} \sigma^{b}=\lambda_{\xi} \lambda_{\sigma}>0$ is the area density of the symmetry $G^{(2)}$-group orbits. In the following we will use the notation $\tau=+\sqrt{\tau^{2}}$. We have now an induced foliation over ${ }^{(2)} \mathcal{M}$ defined by the global time function $t$ introduced before. Let $n^{a}$ be the $g$-unit and future-directed $\left(g^{a b} n_{a}(\mathrm{~d} t)_{b}>0\right)$ vector field normal to this foliation, and let $\hat{\theta}^{a}$ be the $g$-unit spacelike vector field of closed orbits tangent to the slices of constant $t$, such that

$$
\theta^{a}=e^{\gamma / 2} \hat{\theta}^{a}
$$

for some extra field $\gamma$. If we choose the congruence of curves with $t^{a}$ tangent to ${ }^{(2)} \mathcal{M}$. Then, the congruence is transverse to the foliation, and we can express

$$
t^{a}=e^{\gamma / 2}\left(N n^{a}+N^{\theta} \hat{\theta}^{a}\right),
$$

where $N>0$ and $N^{\theta}$ are proportional to the lapse and shift functions. The factor $e^{\gamma / 2}$ will allow us to obtain a proper gauge algebra and simplify later calculations. We require that $N$, $N^{\theta}$, and $\gamma$ are smooth real-valued fields on ${ }^{(3)} \mathcal{M}$. As we will see in the following the symmetry generated by $\sigma^{a}$ will further constraint them, in particular they will be constant along the orbits defined by the remaining Killing vector field. The orthonormal basis $\left(n^{a}, \hat{\theta}^{a}, \sigma^{a} / \tau\right)$ is positively oriented with respect to the volume 3 -form associated to the 3 -metric $g_{a b}$, compatible with $\mathrm{d} t \wedge \mathrm{d} \theta \wedge \mathrm{d} \sigma$, satisfying ${ }^{(3)} \epsilon_{a b c} n^{a} \hat{\theta}^{a} \sigma^{a} / \tau=1$.

The expression of the metric is

$$
g_{a b}=e^{\gamma}\left(\left(N^{\theta 2}-N^{2}\right)(\mathrm{d} t)_{a}(\mathrm{~d} t)_{b}+2 N^{\theta}(\mathrm{d} t)_{(a}(\mathrm{d} \theta)_{b)}+(\mathrm{d} \theta)_{a}(\mathrm{~d} \theta)_{b}\right)+\tau^{2}(\mathrm{~d} \sigma)_{a}(\mathrm{~d} \sigma)_{b} .
$$


The fact that the vectors $\left(t^{a}, \theta^{a}, \sigma^{a}\right)$ commute everywhere will translate into necessary conditions that the vectors $n^{a}$ and $\theta^{a}$ and the scalars $N, N^{\theta}$, and $\gamma$ must satisfy. These are

$$
\begin{gathered}
\mathcal{L}_{\sigma} N=0, \quad \mathcal{L}_{\sigma} N^{\theta}=0, \quad \mathcal{L}_{\sigma} \gamma=0, \\
\left(\mathcal{L}_{\sigma} n\right)^{a}=0, \quad\left(\mathcal{L}_{\sigma} \hat{\theta}\right)^{a}=0
\end{gathered}
$$

and

$$
\frac{1}{2}\left(\mathcal{L}_{\theta} \gamma\right)\left(N n^{a}+N^{\theta} \hat{\theta}^{a}\right)-e^{-\gamma / 2}\left(\mathcal{L}_{t} e^{\gamma / 2}\right) \hat{\theta}^{a}+N e^{\gamma / 2}[\hat{\theta}, n]^{a}+\left(\mathcal{L}_{\theta} N\right) n^{a}+\left(\mathcal{L}_{\theta} N^{\theta}\right) \hat{\theta}^{a}=0 .
$$

This last equation can be projected in the directions defined by the basis vectors to give

$$
\begin{aligned}
& \frac{1}{2} N \mathcal{L}_{\theta} \gamma+\mathcal{L}_{\theta} N+N e^{\gamma / 2} n^{a} n^{b} \nabla_{a} \hat{\theta}_{b}=0, \\
& \frac{1}{2} N^{\theta} \mathcal{L}_{\theta} \gamma+\mathcal{L}_{\theta} N^{\theta}-\frac{1}{2} \mathcal{L}_{t} \gamma+N e^{\gamma / 2} \hat{\theta}^{a} \hat{\theta}^{b} \nabla_{a} n_{b}=0, \\
& \hat{\theta}^{a} \sigma^{b} \nabla_{a} n_{b}=0 .
\end{aligned}
$$

These equations are important because they relate the components of the extrinsic curvature of some surfaces with derivatives of $N, N^{\theta}$, and $\gamma$. Notice that the scalars $\phi_{i}$ are also constant on the orbits of $\sigma^{a}$ (the matter scalar $\phi_{2}$ because we have imposed this from the start and the gravitational scalar $\phi_{1}$ due to the fact that the two Killings $\xi^{a}$ and $\sigma^{a}$ commute: $\mathcal{L}_{\sigma} \lambda_{\xi}=0$ ). Therefore, as we will end up with an essentially two dimensional model with fields depending only on coordinates $t$ and $\theta$, we will eventually denote $\mathcal{L}_{t}$ with a dot and $\mathcal{L}_{\theta}$ with a prime. With this convention, we obtain:

$$
\begin{aligned}
{ }^{(2)} R & =\tau^{-1} e^{-\gamma}\left(\tau^{\prime} \gamma^{\prime}-2 \tau^{\prime \prime}\right), \\
K_{a b} K^{a b}-K^{2} & =-\frac{e^{-\gamma}}{N^{2} \tau}\left(\dot{\gamma}-N^{\theta} \gamma^{\prime}-2 N^{\theta \prime}\right)\left(\dot{\tau}-N^{\theta} \tau^{\prime}\right), \\
g^{a b}\left(\mathrm{~d} \phi_{i}\right)_{a}\left(\mathrm{~d} \phi_{i}\right)_{b} & =-\frac{e^{-\gamma}}{N^{2}}\left(\dot{\phi}_{i}^{2}-2 N^{\theta} \dot{\phi}_{i} \phi_{i}^{\prime}+\left(N^{\theta^{2}}-N^{2}\right) \phi_{i}^{\prime 2}\right),
\end{aligned}
$$

and then the action can be written as

$$
\begin{gathered}
\frac{1}{16 \pi G_{3}} \int_{t_{0}}^{t_{1}} \mathrm{~d} t \int_{\mathbb{T}^{2}}{ }^{(2)} \mathrm{e}|g|^{1 / 2} e^{-\gamma}\left(\frac{1}{\tau}\left(\gamma^{\prime} \tau^{\prime}-2 \tau^{\prime \prime}\right)-\frac{1}{\tau N^{2}}\left(\dot{\gamma}-2 N^{\theta \prime}-\gamma^{\prime} N^{\theta}\right)\left(\dot{\tau}-N^{\theta} \tau^{\prime}\right)\right. \\
\left.+\frac{1}{2 N^{2}} \sum_{i}\left[\dot{\phi}_{i}^{2}-2 N^{\theta} \dot{\phi}_{i} \phi_{i}^{\prime}+\left(N^{\theta 2}-N^{2}\right) \phi_{i}^{\prime 2}\right]\right) .
\end{gathered}
$$

This will be the starting point for the Hamiltonian formalism. Notice that the previous expression is coordinate independent. The Lagrangian is written as an integral over the torus $\mathbb{T}^{2}$ of the 2-form obtained by multiplying the fiducial volume form and a scalar function. All the terms in this scalar are defined through the use of geometrical objects, in particular the derivatives are Lie derivatives along the fields introduced above. This will prove particularly important when dealing with other spatial topologies. In this case, it is natural to choose as fiducial 2-form ${ }^{(2)} \mathrm{e}$ the one verifying ${ }^{(2)} \mathrm{e}_{a b} \theta^{a} \sigma^{b}=N e^{\gamma} \tau /|g|^{1 / 2}=1$, i.e. ${ }^{(2)} \mathrm{e}=\mathrm{d} \theta \wedge \mathrm{d} \sigma$. 
The Hamiltonian can be easily obtained by performing a Legendre transform. It has the form

$$
H=C[N]+C_{\theta}\left[N^{\theta}\right]
$$

where ${ }^{6}$

$$
\begin{aligned}
& C[N]=\int_{\mathbb{S}^{1}} N C:=\int_{\mathbb{S}^{1}} N\left(\frac{1}{8 G_{3}}\left(2 \tau^{\prime \prime}-\gamma^{\prime} \tau^{\prime}\right)-8 G_{3} p_{\gamma} p_{\tau}+\frac{1}{2} \sum_{i}\left(8 G_{3} \frac{p_{\phi_{i}}^{2}}{\tau}+\frac{\tau}{8 G_{3}} \phi_{i}^{\prime 2}\right)\right), \\
& C_{\theta}\left[N^{\theta}\right]=\int_{\mathbb{S}^{1}} N^{\theta} C_{\theta}:=\int_{\mathbb{S}^{1}} N^{\theta}\left(-2 p_{\gamma}^{\prime}+p_{\gamma} \gamma^{\prime}+p_{\tau} \tau^{\prime}+\sum_{i} p_{\phi_{i}} \phi_{i}^{\prime}\right) .
\end{aligned}
$$

The lapse and the shift act as Lagrange multipliers and enforce the constraints $C=0$, $C_{\theta}=0$. The canonical phase space $(\Gamma, \omega)$ is coordinatized by the canonically conjugate pairs $\left(\gamma, p_{\gamma} ; \tau, p_{\tau} ; \phi_{i}, p_{\phi_{i}}\right)$ and $\omega$ denotes the standard (weakly) symplectic form

$$
\omega=\int_{\mathbb{S}^{1}}\left(\delta \gamma \wedge \delta p_{\gamma}+\delta \tau \wedge \delta p_{\tau}+\sum_{i} \delta \phi_{i} \wedge \delta p_{\phi_{i}}\right) .
$$

The dynamical variables are restricted to belong to a constraint submanifold $\Gamma_{c} \subset \Gamma$ globally defined by $C=0, C_{\theta}=0$. The constraints can be written in an equivalent way by taking "linear combinations" obtained by integrating them against suitable weight functions $N_{g}$ and $N_{g}^{\theta}$ in such a way that the vanishing of the weighted form of the constraints for all of them is equivalent to the vanishing of $C$ and $C_{\theta}$ at every point of $\mathbb{S}^{1}$. The gauge transformations generated by the (weighted) constraints are ${ }^{7}$

$$
\begin{aligned}
& \left\{\gamma, C\left[N_{g}\right]\right\}=-N_{g} p_{\tau}, \\
& \left\{\tau, C\left[N_{g}\right]\right\}=-N_{g} p_{\gamma}, \\
& \left\{\phi_{i}, C\left[N_{g}\right]\right\}=N_{g} \frac{p_{\phi_{i}}}{\tau}, \\
& \left\{p_{\gamma}, C\left[N_{g}\right]\right\}=-\left(N_{g} \tau^{\prime}\right)^{\prime}, \\
& \left\{p_{\tau}, C\left[N_{g}\right]\right\}=-\left(N_{g} \gamma^{\prime}\right)^{\prime}+\frac{1}{2} N_{g} \sum_{i}\left(\frac{p_{\phi_{i}}^{2}}{\tau^{2}}-\phi_{i}^{\prime 2}\right), \\
& \left\{p_{\phi_{i}}, C\left[N_{g}\right]\right\}=\left(N_{g} \tau \phi_{i}^{\prime}\right)^{\prime},
\end{aligned}
$$

${ }^{6}$ Here and in the following $\int_{\mathbb{S}^{1}} F:=\int_{\mathbb{S}^{1}} F \mathrm{~d} \theta$.

${ }^{7}$ In the rest of this section we will choose units such that $8 G_{3}=1$. 
and

$$
\begin{aligned}
& \left\{\gamma, C_{\theta}\left[N_{g}^{\theta}\right]\right\}=2 N_{g}^{\theta \prime}+N_{g}^{\theta} \gamma^{\prime}, \\
& \left\{\tau, C_{\theta}\left[N_{g}^{\theta}\right]\right\}=N_{g}^{\theta} \tau^{\prime}, \\
& \left\{\phi_{i}, C_{\theta}\left[N_{g}^{\theta}\right]\right\}=N_{g}^{\theta} \phi_{i}^{\prime}, \\
& \left\{p_{\gamma}, C_{\theta}\left[N_{g}^{\theta}\right]\right\}=\left(N_{g}^{\theta} p_{\gamma}\right)^{\prime}, \\
& \left\{p_{\tau}, C_{\theta}\left[N_{g}^{\theta}\right]\right\}=\left(N_{g}^{\theta} p_{\tau}\right)^{\prime}, \\
& \left\{p_{\phi_{i}}, C_{\theta}\left[N_{g}^{\theta}\right]\right\}=\left(N_{g}^{\theta} p_{\phi_{i}}\right)^{\prime} .
\end{aligned}
$$

A straightforward calculation shows that the constraints are first class in Dirac terminology, or equivalently that $\Gamma_{c}$ is a coisotropic submanifold of $\Gamma$. Indeed, the Poisson algebra of the constraints is a proper Lie algebra

$$
\begin{aligned}
& \left\{C\left[N_{g}\right], C\left[M_{g}\right]\right\}=C_{\theta}\left[N_{g} M_{g}^{\prime}-M_{g} N_{g}^{\prime}\right], \\
& \left\{C\left[N_{g}\right], C_{\theta}\left[N_{g}^{\theta}\right]\right\}=C\left[N_{g} M_{g}^{\theta \prime}-M_{g}^{\theta} N_{g}^{\prime}\right], \\
& \left\{C_{\theta}\left[N_{g}^{\theta}\right], C_{\theta}\left[M_{g}^{\theta}\right]\right\}=C_{\theta}\left[N_{g}^{\theta} M_{g}^{\theta \prime}-M_{g}^{\theta} N_{g}^{\theta \prime}\right] .
\end{aligned}
$$

Notice also that, as a consequence of the introduction of the suitable exponential factor $e^{\gamma / 2}$ in (3.1) we have a closed gauge algebra [12] (i.e. with structure constants).

In order to proceed we would like to isolate the true physical degrees of freedom of the model. As is well known there are several possible ways to do this. The usual ones are gauge fixing, i.e. the isolation of a single point per gauge orbit by imposing appropriate extra conditions on the phase space variables, and phase space reduction -that requires us to find a way to effectively quotient the phase space by the equivalence relation loosely defined as "belonging to the same orbit". The successful implementation of the reduction allows us not only to label gauge orbits but also provides us with important mathematical structures (topological, symplectic,...) from the ones present in the initial phase space. Here we will see that a partial gauge fixing (deparameterization) can provide us with another interesting way to deal with the system because it can be described by a time-dependent, quadratic, Hamiltonian [3, 4, 14]. As we will show below this is also true for the other spatial topologies. If one is interested in quantizing the model one can alternatively use the Hamiltonian formulation described above to attempt a Dirac quantization.

The Hamiltonian vector fields associated to the weighted constraints $C\left[N_{g}\right], C_{\theta}\left[N_{g}^{\theta}\right]$ are tangential to $\Gamma_{c}$ and define the degenerate directions of the presymplectic form $\left.\omega\right|_{\Gamma_{c}}$. The deparameterization procedure is based on the choice of one of these Hamiltonian vector fields to define an evolution vector field $E_{H_{R}}$, generated by a reduced Hamiltonian $H_{R}$ of a generically non-autonomous system. With this aim in mind, we will impose gauge fixing conditions in such a way that at least one of the first class constraints $\mathcal{C}$ is not fixed. This will be used to define dynamics. Any remaining constraints left over by the (partial) gauge fixing will generate residual gauge symmetries.

Let $\iota: \Gamma_{G} \rightarrow \Gamma_{c}$ denote the embedding of the gauge fixed surface given by the first class constraints and the gauge fixing conditions; the pull-back of the presymplectic form to this surface, $\iota^{*} \omega$, has a single degenerate direction defined by the Hamiltonian vector field $E_{H_{R}}$. Select then a suitable phase space variable $T$ such that $E_{H_{R}}(T)=1$. The level surfaces of $T$ 
are all diffeomorphic to a manifold $\Gamma_{R}$ and transverse to $E_{H_{R}}$, defining a foliation of $\Gamma_{G}$ with $T$ as global time function. In that case, $\iota^{*} \omega=-\mathrm{d} T \wedge \mathrm{d} H_{R}+\omega_{R}, E_{H_{R}}=\partial_{T}+X_{H_{R}}$, where $\omega_{R}$ is a weakly non-degenerate form, and $\left(\Gamma_{R}, \omega_{R}, H_{R}(T)\right)$ define a non-autonomous Hamiltonian system. Any remaining first class constraints will define a constrain submanifold in $\Gamma_{R}$.

The conditions that are usually considered for this problem [10, 12, 13, 14] are

$$
\begin{gathered}
\tau^{\prime}=0, \\
p_{\gamma}^{\prime}=0 .
\end{gathered}
$$

They mean that both $\tau$ and $p_{\gamma}$ take the same value irrespective of the point of $\mathbb{S}^{1}$ but they do not specify which one. Notice that conditions of the type $\tau=t_{0}$ or $p_{\gamma}=-p$ with $t_{0}, p \in \mathbb{R}$ not only would tell us that $\tau$ and $p_{\gamma}$ are independent of $\theta$ but also assign a fixed value to them, thus removing additional degrees of freedom. ${ }^{8}$

This means that when using (3.9,3.10) there is still a dynamical mode in $\tau$ that may vary in the evolution -at the end of the day it will be identified with the time parameter- but is constant on every spatial slice in the $3+1$ decomposition. The fact that this class of models have an initial spacetime singularity suggests that there are interesting interpretive issues as far as the equivalence of the different choices of gauge fixing is concerned (how does this singularity manifest itself after a full gauge fixing? how does it show up if other gauge fixing conditions are used?). It should also be pointed out that although it is possible to think of the condition $\tau^{\prime}=0$ as a one parameter family of gauges $\tau=t$, with $t \in(0, \infty)$, it is dangerous to use it in this last form when computing Poisson brackets (it would be something like "mixing parametric and implicit equations") as can be checked by explicit computations. In this case the correct attitude would be to work in the extended ("odd-dimensional") phase space, mathematically described as a cosymplectic or contact manifold, incorporating a time variable and employ the usual techniques for non-autonomous Hamiltonian systems [28, 29].

A convenient way to discuss gauge fixings is to describe our family of gauge conditions by introducing an orthonormal basis of weight functions on $\mathbb{S}^{1}$

$$
Y_{n}(\theta):=\frac{1}{\sqrt{2 \pi}} e^{i n \theta}, \quad n \in \mathbb{Z},
$$

and consider the family of constraints $C\left[Y_{n}\right], C_{\theta}\left[Y_{n}\right]$. By expanding now

$$
\tau=\sum_{n \in \mathbb{Z}} \tau_{n} Y_{n}, \quad p_{\gamma}=\sum_{n \in \mathbb{Z}} p_{\gamma_{n}} Y_{n}
$$

with

$$
\tau_{n}=\frac{1}{\sqrt{2 \pi}} \int_{\mathbb{S}^{1}} e^{-i n \theta} \tau, \quad p_{\gamma_{n}}=\frac{1}{\sqrt{2 \pi}} \int_{\mathbb{S}^{1}} e^{-i n \theta} p_{\gamma},
$$

\footnotetext{
${ }^{8}$ A useful example to appreciate the difference between taking some derivatives to be zero and fixing the values of the functions is to consider the straight line $x_{1}=x_{2}=x_{3}$ in $\mathbb{R}^{3}$ where all the points have equal coordinates in contrast with the point $x_{1}=x_{2}=x_{3}=1$.
} 
the previous gauge fixing conditions become

$$
\tau_{n}=0, \quad p_{\gamma_{n}}=0, \quad \forall n \in \mathbb{Z}-\{0\} .
$$

In order to see if this is a good gauge fixing (and, alternatively, find out if some gauge freedom is left) we compute

$$
\begin{aligned}
& \left\{\tau_{n}, C\left[Y_{m}\right]\right\} \approx-\frac{1}{\sqrt{2 \pi}} \delta_{n m} p_{\gamma_{0}}, \quad\left\{\tau_{n}, C_{\theta}\left[Y_{m}\right]\right\} \approx 0 \\
& \left\{p_{\gamma_{n}}, C\left[Y_{m}\right]\right\} \approx 0, \quad\left\{p_{\gamma n}, C_{\theta}\left[Y_{m}\right]\right\} \approx \frac{i n}{\sqrt{2 \pi}} \delta_{n m} p_{\gamma_{0}}
\end{aligned}
$$

where $n \in \mathbb{Z}-\{0\}, m \in \mathbb{Z}$, and the symbol $\approx$ denotes equality on the hypersurface defined by the gauge fixing conditions and the constraints, the so-called gauge fixing surface $\Gamma_{G} \subset$ $\Gamma_{c}$. Notice that with this way of writing the constraints (without the extra terms that would be present if we had not introduced the exponential prefactor in (3.1)) the gauge transformations of $\tau$ and $p_{\gamma}$ only involve these objects themselves. It is convenient to write the previous expressions in table form

\begin{tabular}{|c|cc|cc|c|}
\hline \hline & $\tau_{1}=0$ & $p_{\gamma_{1}}=0$ & $\tau_{-1}=0$ & $p_{\gamma_{-1}}=0$ & $\ldots$ \\
\hline$C\left[Y_{0}\right]$ & 0 & 0 & 0 & 0 & $\ldots$ \\
$C_{\theta}\left[Y_{0}\right]$ & 0 & 0 & 0 & 0 & $\ldots$ \\
\hline$C\left[Y_{1}\right]$ & $-\frac{1}{\sqrt{2 \pi}} p_{\gamma 0}$ & 0 & 0 & 0 & $\ldots$ \\
$C_{\theta}\left[Y_{1}\right]$ & 0 & $\frac{i}{\sqrt{2 \pi}} p_{\gamma 0}$ & 0 & 0 & $\ldots$ \\
\hline$C\left[Y_{-1}\right]$ & 0 & 0 & $-\frac{1}{\sqrt{2 \pi}} p_{\gamma 0}$ & 0 & $\ldots$ \\
$C_{\theta}\left[Y_{-1}\right]$ & 0 & 0 & 0 & $-\frac{i}{\sqrt{2 \pi}} p_{\gamma 0}$ & $\ldots$ \\
\hline$\vdots$ & $\vdots$ & $\vdots$ & $\vdots$ & $\vdots$ & $\ddots$ \\
\hline \hline
\end{tabular}

As we can see the only constraints that are not gauge-fixed by the conditions introduced above, as long as $p_{\gamma_{0}} \neq 0$, are $C[1]=0$ and $C_{\theta}[1]=0$. From now on we will consider the sector $p_{\gamma_{0}}<0$. As we can see we have two first class constraints left over by our partial gauge fixing

$$
\begin{aligned}
& \int_{\mathbb{S}^{1}}\left(\left(2 \tau^{\prime \prime}-\gamma^{\prime} \tau^{\prime}\right)-p_{\gamma} p_{\tau}+\frac{1}{2} \sum_{i}\left(\frac{p_{\phi_{i}}^{2}}{\tau}+\tau \phi_{i}^{\prime 2}\right)\right) \approx 0, \\
& \int_{\mathbb{S}^{1}}\left(-2 p_{\gamma}^{\prime}+p_{\gamma} \gamma^{\prime}+p_{\tau} \tau^{\prime}+\sum_{i} p_{\phi_{i}} \phi_{i}^{\prime}\right) \approx 0 .
\end{aligned}
$$

We can pullback the relevant geometric objects to the submanifold $\Gamma_{G}$ defined by the gauge fixing conditions to eliminate some of the variables in our model. Denoting by $\iota: \Gamma_{G} \rightarrow \Gamma$ the immersion map, the pullback of the (weakly) symplectic form (3.8) becomes

$$
\iota^{*} \omega=\mathrm{d} \gamma_{0} \wedge \mathrm{d} p_{\gamma_{0}}+\mathrm{d} \tau_{0} \wedge \mathrm{d} p_{\tau_{0}}+\sum_{i} \int_{\mathbb{S}^{1}} \delta \phi_{i} \wedge \delta p_{\phi_{i}} .
$$


The pullback of the constraints (3.12,3.13) is

$$
\begin{aligned}
& \mathcal{C}:=-p_{\gamma_{0}} p_{\tau_{0}}+\frac{1}{2} \sum_{i} \int_{\mathbb{S}^{1}}\left(\sqrt{2 \pi} \frac{p_{\phi_{i}}^{2}}{\tau_{0}}+\frac{\tau_{0}}{\sqrt{2 \pi}} \phi_{i}^{\prime 2}\right) \approx 0, \\
& \mathcal{C}_{\theta}:=\sum_{i} \int_{\mathbb{S}^{1}} p_{\phi_{i}} \phi_{i}^{\prime} \approx 0 .
\end{aligned}
$$

Let us look now at the gauge transformations of $\tau_{0}$ generated by (3.15)

$$
\begin{aligned}
& \left\{\tau_{0}, \mathcal{C}\right\} \approx-p_{\gamma_{0}}, \\
& \left\{p_{\gamma_{0}}, \mathcal{C}\right\} \approx 0 .
\end{aligned}
$$

If $s \in(0, \infty)$ parameterizes the gauge orbits we see that on them we have $\tau_{0}=p s$ and $p_{\gamma_{0}}=-p$, with $p>0$. This suggests that a simplification of our model will occur if we introduce a canonical transformation where $\tau_{0}$ and $p_{\gamma_{0}}$ are substituted for new canonical variables. Indeed, the canonical transformation [6]

$$
\begin{aligned}
\tau_{0} & =T P, \quad p_{\tau_{0}}=\frac{p_{T}}{P}, \\
\gamma_{0}=-\frac{1}{\sqrt{2 \pi}}\left(Q+\frac{p_{T}}{P} T\right), p_{\gamma_{0}} & =-\sqrt{2 \pi} P,
\end{aligned}
$$

with $(Q, P>0)$, and $\left(T, p_{T}\right)$ canonically conjugate pairs, allows us to write

$$
\mathcal{C}=p_{T}+\frac{1}{2} \sum_{i} \int_{\mathbb{S}^{1}}\left(\frac{p_{\phi_{i}}^{2}}{P T}+P T \phi_{i}^{\prime 2}\right) \approx 0 .
$$

Finally the canonical transformation (here $(\tilde{Q}, \tilde{P})$ and $\left(\varphi_{i}, p_{\varphi_{i}}\right)$ are new canonical pairs [6])

$$
\begin{aligned}
\tilde{Q} & :=P Q+\frac{1}{2} \sum_{i} \int_{\mathbb{S}^{1}} p_{\phi_{i}} \phi_{i}, \quad \tilde{P}:=\log P \\
\varphi_{i} & :=\sqrt{P} \phi_{i}, \quad p_{\varphi_{i}}:=\frac{1}{\sqrt{P}} p_{\phi_{i}},
\end{aligned}
$$

turns the constraints (3.17,3.16) into

$$
\begin{aligned}
& \mathcal{C}=p_{T}+\frac{1}{2} \sum_{i} \int_{\mathbb{S}^{1}}\left(\frac{p_{\varphi_{i}}^{2}}{T}+T \varphi_{i}^{\prime 2}\right) \approx 0 \\
& \mathcal{C}_{\theta}=\sum_{i} \int_{\mathbb{S}^{1}} p_{\varphi_{i}} \varphi_{i}^{\prime} \approx 0
\end{aligned}
$$

and the 2-form (3.14) becomes

$$
\iota^{*} \omega=\mathrm{d} \tilde{Q} \wedge \mathrm{d} \tilde{P}+\sum_{i} \int_{\mathbb{S}^{1}} \delta \varphi_{i} \wedge \delta p_{\varphi_{i}}+\mathrm{d} T \wedge \mathrm{d} p_{T} .
$$


The fact that (3.19) is linear in $p_{T}$ allows us to interpret the 4-tuple $\left((0, \infty) \times \Gamma_{R}, \mathrm{~d} t, \omega_{R}, H_{R}\right)$ as a non-autonomous Hamiltonian system with $T=t$ as the time parameter, restricted to verify the global constraint (3.20). The reduced phase space $\Gamma_{R}$ is coordinatized now by the canonical pairs $\left(\tilde{Q}, \tilde{P} ; \varphi_{i}, p_{\varphi_{i}}\right)$ and is endowed with the (weakly) symplectic form

$$
\omega_{R}:=\mathrm{d} \tilde{Q} \wedge \mathrm{d} \tilde{P}+\sum_{i} \int_{\mathbb{S}^{1}} \delta \varphi_{i} \wedge \delta p_{\varphi_{i}} .
$$

The reduced time-dependent Hamiltonian $H_{R}(t): \Gamma_{R} \rightarrow \mathbb{R}$ is given by

$$
H_{R}(t)=\frac{1}{2} \sum_{i} \int_{\mathbb{S}^{1}}\left(\frac{p_{\varphi_{i}}^{2}}{t}+t \varphi_{i}^{\prime 2}\right),
$$

and the evolution vector field is given by

$$
E_{H_{R}}=\frac{\partial}{\partial t}+\sum_{i} \int_{\mathbb{S}^{1}}\left(\frac{p_{\varphi_{i}}}{t} \frac{\delta}{\delta \varphi_{i}}+t \varphi_{i}^{\prime \prime} \frac{\delta}{\delta p_{\varphi_{i}}}\right) .
$$

This defines the only degenerate direction of (3.21). Although the form of the Hamiltonian that we have just obtained seems to suggest that the gravitational and matter scalars are not coupled, in fact the constraint (3.20) shows that this is not the case ${ }^{9}$. Notice also that the canonical pair $(\tilde{Q}, \tilde{P})$ describes a global degree of freedom even though they are constants of motion under the dynamics generated by (3.23). The singularities that must be present in this case as a consequence of the Hawking-Penrose theorems [25] can be understood as coming from the singular behavior at $t=0$ of the Hamiltonian (3.23).

Finally, it is possible to recover the original 4-dimensional spacetime from this 3dimensional formulation. First notice that the gauge fixing conditions defining the deparameterization are preserved under the dynamics if and only if the lapse and shift functions $N$ and $N^{\theta}$ are constant. By redefining the coordinate $\theta$ as in [9] we can eliminate the shift function from the metric. We can proceed in an analogous way for the lapse function to make it equal to 1 . Once we integrate the Hamiltonian equations corresponding to (3.23), undo the canonical transformation defined above, and solve the constraint $C_{\theta}=0$ in order to obtain the $\gamma$ function, we uniquely determine the 3-metric (3.2), and hence the original 4-metric.

\section{IV. $\mathbb{S}^{1} \times \mathbb{S}^{2}$ GOWDY MODELS COUPLED TO MASSLESS SCALARS}

Let us consider now the three-handle ${ }^{(3)} \Sigma=\mathbb{S}^{1} \times \mathbb{S}^{2}$, parameterized as $\left(e^{i \xi}, e^{i \sigma} \sin \theta, \cos \theta\right)$ with $\theta \in[0, \pi], \xi, \sigma \in \mathbb{R}(\bmod 2 \pi)$. Using the group parametrization introduced above we can write the $G^{(2)}$-group action in the form

$$
\left(g_{1}, g_{2}\right) \cdot\left(e^{i \xi}, e^{i \sigma} \sin \theta, \cos \theta\right)=\left(e^{x_{1}}, e^{x_{2}}\right) \cdot\left(e^{i \xi}, e^{i \sigma} \sin \theta, \cos \theta\right)=\left(e^{i\left(x_{1}+\xi\right)}, e^{i\left(x_{2}+\sigma\right)} \sin \theta, \cos \theta\right) .
$$

\footnotetext{
9 The matter fields act as sources for the gravitational field, hence, the solutions to the Einstein equations should depend on the matter content.
} 
The action of the two $U(1)$ subgroup factors of $G^{(2)}$ is

$$
\begin{aligned}
& \left(1, e^{i x}\right) \cdot\left(e^{i \xi}, e^{i \sigma} \sin \theta, \cos \theta\right)=\left(e^{i \xi}, e^{i(x+\sigma)} \sin \theta, \cos \theta\right), \\
& \left(e^{i x}, 1\right) \cdot\left(e^{i \xi}, e^{i \sigma} \sin \theta, \cos \theta\right)=\left(e^{i(x+\xi)}, e^{i \sigma} \sin \theta, \cos \theta\right) .
\end{aligned}
$$

The corresponding tangent vectors at each point of ${ }^{(3)} \Sigma$, obtained by differentiating the previous expressions with respect to $x$ at $x=0$, are

$$
\begin{aligned}
& \left(0, i e^{i \sigma} \sin \theta, 0\right), \\
& \left(i e^{i \xi}, 0,0\right) .
\end{aligned}
$$

As we can see the second one is never zero but the first one vanishes at the poles of the sphere $\mathbb{S}^{2}$ where $\theta=0, \pi$. This corresponds to the circumferences given by $\left(e^{i \xi}, 0,1\right)$ and $\left(e^{i \xi}, 0,-1\right)$. It is straightforward to verify that both fields commute. In view of all this we perform a Geroch reduction by using the non-vanishing Killing. After a suitable conformal transformation the field equations can be derived from an action of the form (2.4) with ${ }^{(2)} \Sigma=\mathbb{S}^{2}$. All the fields in this action are defined on $\mathbb{S}^{2}$ and are symmetric under the symmetries generated by the remaining Killing $\sigma^{a}$. Since this Killing vector vanishes at the poles of the sphere $\mathbb{S}^{2}$ we cannot build an everywhere orthonormal basis that involves this vector. In fact, we know that as $\mathbb{S}^{2}$ is not paralelizable this is impossible on general grounds. We nevertheless will consider the triplet of vectors $\left(n^{a}, \hat{\theta}^{a}, \sigma^{a} / \tau\right)$ whenever it is different from zero (for all $\theta \neq 0, \pi$ ). Taking again the definition (3.1), the form of the metric is the same as in the $\mathbb{T}^{3}$ case (3.2). The symmetry of the problem implies also that $\left(N, N^{\theta}, \gamma, \tau, \phi_{i}\right)$ are constant on the orbits of the Killing field $\sigma^{a}$.

A very important issue now is the regularity of the metric. From a classical point of view the final outcome of the Hamiltonian analysis of the system is a set of equations whose solutions allow us to reconstruct a four dimensional spacetime metric and a set of scalar fields satisfying the coupled Einstein-Klein Gordon equations. This means that once we decide the functional space to which this metric belongs this will imply that the objects that appear during the dimensional reduction, gauge fixing and so on may be subject to some regularity conditions. In the $\mathbb{T}^{3}$ case these are simple smoothness requirements but in the present case, due to the existence of a symmetry axis, these are more complicated. The regularity conditions that the metric components for an axially symmetric metric must verify can be deduced as in [23, 27]. By using the coordinates $(t, \theta, \sigma, \xi)$, we can write the original 4-metric ${ }^{(4)} g_{a b}$ as

$$
\begin{aligned}
{ }^{(4)} g_{a b} & =e^{\left(\gamma-\phi_{1}\right)}\left[\left(N^{\theta 2}-N^{2}\right)(\mathrm{d} t)_{a}(\mathrm{~d} t)_{b}+2 N^{\theta}(\mathrm{d} t)_{(a}(\mathrm{d} \theta)_{b)}+(\mathrm{d} \theta)_{a}(\mathrm{~d} \theta)_{b}\right] \\
& +\tau^{2} e^{-\phi_{1}}(\mathrm{~d} \sigma)_{a}(\mathrm{~d} \sigma)_{b}+e^{\phi_{1}}(\mathrm{~d} \xi)_{a}(\mathrm{~d} \xi)_{b} .
\end{aligned}
$$

This means that we have the following regularity conditions for $\theta \rightarrow 0, \pi$ (if we impose analyticity, otherwise we need only to know the asymptotic behavior for small values of 
$\sin \theta)$

$$
\begin{aligned}
& e^{\left(\gamma-\phi_{1}\right)}\left(N^{\theta 2}-N^{2}\right)=A(t, \cos \theta), \\
& e^{\left(\gamma-\phi_{1}\right)} N^{\theta}=B(t, \cos \theta) \sin \theta, \\
& e^{\phi_{1}}=C(t, \cos \theta), \\
& e^{\gamma-\phi_{1}}=D(t, \cos \theta)+E(t, \cos \theta) \sin ^{2} \theta, \\
& \tau^{2} e^{-\phi_{1}}=\sin ^{2} \theta\left[D(t, \cos \theta)-E(t, \cos \theta) \sin ^{2} \theta\right],
\end{aligned}
$$

where $A, B, C, D, E$ are analytic in their arguments (despite of the fact that they also depend on $t$, as we will use them in the Hamiltonian formulation of the model we will not write the $t$ dependence explicitly in the following). Notice, in particular, that the functions $D$ and $E$ both appear in the last two expressions. The conditions for the fields themselves (dropping the $t$ dependence) are

$$
\begin{aligned}
& \phi_{i}=\hat{\phi}_{i}(\cos \theta), \\
& \gamma=\hat{\gamma}(\cos \theta), \\
& N^{\theta}=\hat{N}^{\theta}(\cos \theta) \sin \theta, \\
& N=\hat{N}(\cos \theta), \\
& \tau=\hat{T}(\cos \theta) \sin \theta, \\
& \tau^{2} e^{-\gamma}=\frac{D(\cos \theta)-E(\cos \theta) \sin ^{2} \theta}{D(\cos \theta)+E(\cos \theta) \sin ^{2} \theta} \sin ^{2} \theta,
\end{aligned}
$$

where $\hat{\phi}_{i}, \hat{\gamma}, \hat{N}^{\theta}, \hat{N}, \hat{T}:[-1,1] \rightarrow \mathbb{R}(\hat{N}>0)$ and can be written as functions of $A, B, C, D, E$. They must be differentiable in $(-1,1)$ and their right and left derivatives at \pm 1 must be defined (equivalently they must be $\mathcal{C}^{\infty}$ in $(-1,1)$ with bounded derivative). Several comments are in order now. First we have been able to write all the relevant fields in such a way that their singular dependence has been factored out $(\sin \theta$ is not a smooth function on the sphere). The functions defined on $\mathbb{S}^{2}$ as $\hat{\phi}_{i} \circ \cos \theta, \hat{\gamma} \circ \cos \theta, \hat{N}^{\theta} \circ \cos \theta, \hat{N} \circ \cos \theta, \hat{T} \circ \cos \theta$ can be alternatively viewed as analytic functions on the sphere invariant under rotations around its symmetry axis and can be considered as the basic fields to describe our system. In fact we will do so in the following. We will refer to these functions on the sphere as $\hat{\phi}_{i}, \hat{\gamma}, \hat{N}^{\theta}, \hat{N}, \hat{T}$ (without the o $\cos \theta$ that will only be used if the possibility of confusion arises) and collectively as the hat-fields. In the following we will write everything in terms of them. Second we can see that condition (4.12) implies that

$$
\hat{T}( \pm 1)=e^{\hat{\gamma}( \pm 1) / 2}
$$

This means that the values of the fields $\hat{T}$ and $\hat{\gamma}$ at the poles of the sphere are not independent of each other. This is a new feature, not present for the $\mathbb{T}^{3}$ topology, that must be taken into account. As we will see these are necessary ingredients to guarantee the consistency of the model.

Given a smooth (and axially symmetric) function on $\mathbb{S}^{2}$ its Lie derivative along $\theta^{a}, \mathcal{L}_{\theta}$, cannot necessarily be extended as a smooth function on the sphere. The function $\cos \theta$ itself is an example of this because $\mathcal{L}_{\theta} \cos \theta=-\sin \theta$. We can, however, define a smooth derivative $f^{\prime}$ for a smooth axially symmetric function as the extension of $f^{\prime}:=-\frac{1}{\sin \theta} \partial_{\theta} f$ to $\mathbb{S}^{2}$ (this is 
formally done by considering $f$ as a function of $\cos \theta$ and differentiating). In the following the prime symbol will always refer to this derivative.

It is natural to consider in this case ${ }^{(2)} \mathrm{e}$ as the fiducial 2 -form associated to a round metric, such that ${ }^{(2)} \mathrm{e}_{a b} \theta^{a} \sigma^{b}=\hat{N} e^{\hat{\gamma}} \hat{T} \sin \theta /|g|^{1 / 2}=\sin \theta$, i.e. ${ }^{(2)} \mathrm{e}=\sin \theta \mathrm{d} \theta \wedge \mathrm{d} \sigma$. Taking this into account we get an action

$$
\begin{aligned}
\frac{1}{16 \pi G_{3}} \int_{t_{0}}^{t_{1}} \mathrm{~d} t \int_{\mathbb{S}^{2}}{ }^{(2)} \mathrm{e} & {\left[\hat{N}\left[\left(\hat{\gamma}^{\prime} \hat{T}^{\prime}-2 \hat{T}^{\prime \prime}\right) \sin ^{2} \theta+\left(6 \hat{T}^{\prime}-\hat{\gamma}^{\prime} \hat{T}\right) \cos \theta+2 \hat{T}\right]\right.} \\
& +\frac{1}{\hat{N}}\left[\hat{N}^{\theta} \hat{T} \cos \theta-\dot{\hat{T}}-\hat{N}^{\theta} \hat{T}^{\prime} \sin ^{2} \theta\right]\left[\dot{\hat{\gamma}}+\left(2 \hat{N}^{\theta \prime}+\hat{N}^{\theta} \hat{\gamma}^{\prime}\right) \sin ^{2} \theta-2 \hat{N}^{\theta} \cos \theta\right] \\
& \left.+\frac{\hat{T}}{2 \hat{N}} \sum_{i}\left(\dot{\hat{\phi}}_{i}^{2}+2 \hat{N}^{\theta} \dot{\hat{\phi}}_{i} \hat{\phi}_{i}^{\prime} \sin ^{2} \theta+\left(\hat{N}^{\theta 2} \sin ^{2} \theta-\hat{N}^{2}\right) \hat{\phi}_{i}^{\prime 2} \sin ^{2} \theta\right)\right] .
\end{aligned}
$$

As we can see it is expressed as the integral of a smooth function on the sphere. This is so because all the fields that appear in the integrand are either the hat-fields, their prime derivatives or smooth functions of $\cos \theta$. The Hamiltonian can be readily derived from the previous action and, as in the $\mathbb{T}^{3}$ case, is of the form $H=C[\hat{N}]+C_{\theta}\left[\hat{N}^{\theta}\right]$ with

$$
\begin{aligned}
C[\hat{N}]= & \int_{\mathbb{S}^{2}}{ }^{(2)} \mathrm{e} \hat{N} C \\
:= & \int_{\mathbb{S}^{2}}{ }^{(2)} \mathrm{e} \hat{N}\left[-16 \pi G_{3} p_{\hat{\gamma}} p_{\hat{T}}+\frac{1}{16 \pi G_{3}}\left[\left(2 \hat{T}^{\prime \prime}-\hat{\gamma}^{\prime} \hat{T}^{\prime}\right) \sin ^{2} \theta+\left(\hat{\gamma}^{\prime} \hat{T}-6 \hat{T}^{\prime}\right) \cos \theta-2 \hat{T}\right]\right. \\
& \left.\quad+\frac{1}{2} \sum_{i}\left(\frac{16 \pi G_{3}}{\hat{T}} p_{\hat{\phi}_{i}}^{2}+\frac{\hat{T}}{16 \pi G_{3}} \hat{\phi}_{i}^{\prime 2} \sin ^{2} \theta\right)\right] \\
C_{\theta}\left[\hat{N}^{\theta}\right]= & \int_{\mathbb{S}^{2}}{ }^{(2)} \mathrm{e} \hat{N}^{\theta} C_{\theta} \\
:= & \int_{\mathbb{S}^{2}}^{(2)} \mathrm{e} \hat{N}^{\theta}\left(\left[2 p_{\hat{\gamma}}^{\prime}-\hat{\gamma}^{\prime} p_{\hat{\gamma}}-\hat{T}^{\prime} p_{\hat{T}}-\sum_{i} \hat{\phi}_{i}^{\prime} p_{\hat{\phi}_{i}}\right] \sin ^{2} \theta+\left[\hat{T} p_{\hat{T}}-2 p_{\hat{\gamma}}\right] \cos \theta\right) .
\end{aligned}
$$

Again, the dynamical variables are restricted to belong to a constraint surface $\Gamma_{c} \subset \Gamma$ in the canonical phase space of the system $(\Gamma, \omega)$, globally defined by the constraints $C=0$, $C_{\theta}=0 . \Gamma$ is coordinatized by the conjugated pairs $\left(\hat{\gamma}, p_{\hat{\gamma}} ; \hat{T}, p_{\hat{T}} ; \hat{\phi}_{i}, p_{\hat{\phi}_{i}}\right)$ and endowed with the standard (weakly) symplectic form

$$
\omega:=\int_{\mathbb{S}^{2}}{ }^{(2)} \mathrm{e}\left(\delta \hat{\gamma} \wedge \delta p_{\hat{\gamma}}+\delta \hat{T} \wedge \delta p_{\hat{T}}+\sum_{i} \delta \hat{\phi}_{i} \wedge \delta p_{\hat{\phi}_{i}}\right) .
$$


The gauge transformations generated by these constraints $\operatorname{are}^{10}$

$$
\begin{aligned}
\left\{\hat{\gamma}, C\left[\hat{N}_{g}\right]\right\}= & -\hat{N}_{g} p_{\hat{T}}, \\
\left\{\hat{T}, C\left[\hat{N}_{g}\right]\right\}= & -\hat{N}_{g} p_{\hat{\gamma}}, \\
\left\{\hat{\phi}_{i}, C\left[\hat{N}_{g}\right]\right\}= & \frac{\hat{N}^{g}}{\hat{T}} p_{\hat{\phi}_{i}}, \\
\left\{p_{\hat{\gamma}}, C\left[\hat{N}_{g}\right]\right\}= & \hat{N}_{g}^{\prime}\left(\hat{T} \cos \theta-\hat{T}^{\prime} \sin ^{2} \theta\right)+\hat{N}_{g}\left(\hat{T}+3 \hat{T}^{\prime} \cos \theta-\hat{T}^{\prime \prime} \sin ^{2} \theta\right), \\
\left\{p_{\hat{T}}, C\left[\hat{N}_{g}\right]\right\}= & \hat{N}_{g}^{\prime}\left(2 \cos \theta-\hat{\gamma}^{\prime} \sin ^{2} \theta\right)+\hat{N}_{g}\left(\hat{\gamma}^{\prime} \cos \theta-\hat{\gamma}^{\prime \prime} \sin ^{2} \theta\right)-2 \hat{N}_{g}^{\prime \prime} \sin ^{2} \theta \\
& +\frac{\hat{N}_{g}}{2} \sum_{i}\left(\frac{p_{\hat{\phi}_{i}}^{2}}{\hat{T}^{2}}-\sin ^{2} \theta \hat{\phi}_{i}^{\prime 2}\right), \\
\left\{p_{\hat{\phi}_{i}}, C\left[\hat{N}_{g}\right]\right\}= & \hat{N}_{g}^{\prime} \hat{T} \hat{\phi}_{i}^{\prime} \sin ^{2} \theta+\hat{N}_{g}\left[\left(\hat{T}^{\prime} \hat{\phi}_{i}^{\prime}+\hat{T} \hat{\phi}_{i}^{\prime \prime}\right) \sin ^{2} \theta-2 \hat{T} \hat{\phi}_{i}^{\prime} \cos \theta\right],
\end{aligned}
$$

and

$$
\begin{aligned}
& \left\{\hat{\gamma}, C_{\theta}\left[\hat{N}_{g}^{\theta}\right]\right\}=-2 \hat{N}_{g}^{\theta \prime} \sin ^{2} \theta+\hat{N}_{g}^{\theta}\left(2 \cos \theta-\hat{\gamma}^{\prime} \sin ^{2} \theta\right), \\
& \left\{\hat{T}, C_{\theta}\left[\hat{N}_{g}^{\theta}\right]\right\}=\hat{N}_{g}^{\theta}\left(\hat{T} \cos \theta-\hat{T}^{\prime} \sin ^{2} \theta\right), \\
& \left\{\hat{\phi}_{i}, C_{\theta}\left[\hat{N}_{g}^{\theta}\right]\right\}=-\hat{N}_{g}^{\theta} \hat{\phi}_{i}^{\prime} \sin ^{2} \theta, \\
& \left\{p_{\hat{\gamma}}, C_{\theta}\left[\hat{N}_{g}^{\theta}\right]\right\}=\hat{N}_{g}^{\theta}\left(2 p_{\hat{\gamma}} \cos \theta-p_{\hat{\gamma}}^{\prime} \sin ^{2} \theta\right)-\hat{N}_{g}^{\theta \prime} p_{\hat{\gamma}} \sin ^{2} \theta, \\
& \left\{p_{\hat{T}}, C_{\theta}\left[\hat{N}_{g}^{\theta}\right]\right\}=\hat{N}_{g}^{\theta}\left(p_{\hat{T}} \cos \theta-p_{\hat{T}^{\prime}}^{\prime} \sin ^{2} \theta\right)-\hat{N}_{g}^{\theta \prime} p_{\hat{T}} \sin ^{2} \theta, \\
& \left\{p_{\hat{\phi}_{i}}, C_{\theta}\left[\hat{N}_{g}^{\theta}\right]\right\}=\hat{N}_{g}^{\theta}\left(2 p_{\hat{\phi}_{i}} \cos \theta-p_{\hat{\phi}_{i}}^{\prime} \sin ^{2} \theta\right)-\hat{N}_{g}^{\theta \prime} p_{\hat{\phi}_{i}} \sin ^{2} \theta .
\end{aligned}
$$

As is the $\mathbb{T}^{3}$ case, $\Gamma_{c} \subset \Gamma$ is a first class submanifold as can be seen by computing the Poisson algebra of the (weighted) constraints

$$
\begin{aligned}
& \left\{C\left[\hat{N}_{g}\right], C\left[\hat{M}_{g}\right]\right\}=C_{\theta}\left[\hat{M}_{g} \hat{N}_{g}^{\prime}-\hat{N}_{g} \hat{M}_{g}^{\prime}\right], \\
& \left\{C\left[\hat{N}_{g}\right], C_{\theta}\left[\hat{M}_{g}^{\theta}\right]\right\}=C\left[\left(\hat{M}_{g}^{\theta} \hat{N}_{g}^{\prime}-\hat{N}_{g} \hat{M}_{g}^{\theta \prime}\right) \sin ^{2} \theta+\hat{N}_{g} \hat{M}_{g}^{\theta} \cos \theta\right], \\
& \left\{C_{\theta}\left[\hat{N}_{g}^{\theta}\right], C_{\theta}\left[\hat{M}_{g}^{\theta}\right]\right\}=C_{\theta}\left[\left(\hat{M}_{g}^{\theta} \hat{N}_{g}^{\theta \prime}-\hat{N}_{g}^{\theta} \hat{M}_{g}^{\theta \prime}\right) \sin ^{2} \theta\right] .
\end{aligned}
$$

We want to check now the stability of the "polar constraints" $\left(\hat{T} e^{-\hat{\gamma} / 2}\right)( \pm 1)=1$. To this end we compute

$$
\begin{aligned}
& \left\{\hat{T} e^{-\hat{\gamma} / 2}, C\left[\hat{N}_{g}\right]\right\}=8 \pi G_{3} \hat{N}_{g} e^{-\hat{\gamma} / 2}\left(\hat{T} p_{\hat{T}}-2 p_{\hat{\gamma}}\right), \\
& \left\{\hat{T} e^{-\hat{\gamma} / 2}, C_{\theta}\left[\hat{N}_{g}^{\theta}\right]\right\}=e^{-\hat{\gamma} / 2}\left[\hat{T} \hat{N}_{g}^{\theta \prime}+\hat{N}_{g}^{\theta}\left(\frac{1}{2} \hat{T} \hat{\gamma}^{\prime}-\hat{T}^{\prime}\right)\right] \sin ^{2} \theta .
\end{aligned}
$$

The first expression vanishes at the poles as a consequence of the constraint (4.15) for $\theta=0, \pi(\sin \theta=0$ and $|\cos \theta|=1)$ whereas the second vanishes because of the $\sin ^{2} \theta$

${ }^{10}$ In the following $16 \pi G_{3}=1$. 
factor. We then conclude that there are no secondary constraints coming from the stability of the polar constraints. An interesting point to highlight here is the fact that these polar constraints are necessary conditions for the differentiability of the constraints (4.14,4.15).

Deparameterization in this case is carried out by basically following the same steps as in the $\mathbb{T}^{3}$ case. Again, in view of the gauge transformations, we begin by choosing gauge fixing conditions similar to (3.93.10)

$$
\begin{aligned}
& \hat{T}^{\prime}=0, \\
& p_{\hat{\gamma}}^{\prime}=0 .
\end{aligned}
$$

We introduce now an orthonormal basis of functions on the subspace of axially symmetric functions on $\mathbb{S}^{2}$

$$
Y_{n}(\theta)=\left(\frac{2 n+1}{4 \pi}\right)^{1 / 2} P_{n}(\cos \theta), n \in \mathbb{N} \cup\{0\},
$$

where $P_{n}$ are the Legendre polynomials. By expanding now

$$
\hat{T}=\sum_{n=0}^{\infty} \hat{T}_{n} Y_{n}, \quad p_{\hat{\gamma}}=\sum_{n=0}^{\infty} p_{\hat{\gamma}_{n}} Y_{n}
$$

with

$$
\hat{T}_{n}=\left(\frac{2 n+1}{4 \pi}\right)^{1 / 2} \int_{\mathbb{S}^{2}}^{(2)} \mathrm{e} P_{n}(\cos \theta) \hat{T}, \quad p_{\hat{\gamma}_{n}}=\left(\frac{2 n+1}{4 \pi}\right)^{1 / 2} \int_{\mathbb{S}^{2}}{ }^{(2)} \mathrm{e} P_{n}(\cos \theta) p_{\hat{\gamma}},
$$

the conditions (4.17]4.18) become

$$
\hat{T}_{n}=0=p_{\hat{\gamma}_{n}}, \quad \forall n \in \mathbb{N}
$$

In order to see if this is a good gauge fixing (and, alternatively, find out if some gauge 
freedom is left) we compute

$$
\begin{aligned}
& \left\{\hat{T}_{n}, C\left[Y_{m}\right]\right\} \approx-\sqrt{\frac{(2 n+1)(2 m+1)}{(4 \pi)^{3}}} p_{\hat{\gamma} 0} \int_{\mathbb{S}^{2}}^{(2)} \mathrm{e} P_{n}(\cos \theta) P_{m}(\cos \theta)=\frac{1}{\sqrt{4 \pi}} p_{\hat{\gamma} 0} \delta_{n m} \\
& \left\{\hat{T}_{n}, C_{\theta}\left[Y_{m}\right]\right\} \approx \frac{\hat{T}_{0}}{4 \pi} \sqrt{\frac{(2 n+1)(2 m+1)}{4 \pi}} \int_{\mathbb{S}^{2}}{ }^{(2)} \mathrm{e} \cos \theta P_{n}(\cos \theta) P_{m}(\cos \theta) \\
& = \begin{cases}-\frac{(m+1) \hat{T}_{0}}{\sqrt{4 \pi(2 m+1)(2 m+3)}} & \text { if } n=m+1 \\
-\frac{m \hat{T}_{0}}{\sqrt{4 \pi(2 m+1)(2 m-1)}} & \text { if } n=m-1 \\
0 \quad \text { otherwise } & \end{cases} \\
& \left\{p_{\gamma n}, C\left[Y_{m}\right]\right\} \approx \hat{T}_{0} \sqrt{\frac{(2 n+1)(2 m+1)}{(4 \pi)^{3}}} \int_{\mathbb{S}^{2}}{ }^{(2)} \mathrm{e} P_{n}(\cos \theta)\left[P_{m}(\cos \theta)+\cos \theta P_{m}^{\prime}(\cos \theta)\right] \\
& =\left\{\begin{array}{l}
0 \quad \text { if } m=0 \text { or } m<n \\
-\hat{T}_{0} \frac{n+1}{\sqrt{4 \pi}} \quad \text { if } m=n \\
\star \quad \text { otherwise }
\end{array}\right. \\
& \left\{p_{\gamma n}, C_{\theta}\left[Y_{m}\right]\right\} \approx p_{\gamma 0} \sqrt{\frac{(2 n+1)(2 m+1)}{(4 \pi)^{3}}} \int_{\mathbb{S}^{2}}^{(2)} \mathrm{e} P_{n}(\cos \theta)\left[P_{m}(\cos \theta)-\sin ^{2} \theta P_{m}^{\prime}(\cos \theta)\right] \\
& = \begin{cases}0 & \text { if } m=0 \text { or } m<n-1 \\
* & \text { otherwise }\end{cases}
\end{aligned}
$$

where the symbol $\approx$ denotes that we are restricting ourselves to points in the hypersurface $\Gamma_{G} \subset \Gamma_{c}$ defined by the gauge fixing conditions and the constraints. The $\star$ and $*$ symbols denote terms (computable in closed form but with somewhat complicated expressions) that are not needed in the following discussion. As before it helps to display the previous result in table form

\begin{tabular}{|c|cc|cc|c|}
\hline \hline & $\hat{T}_{1}=0$ & $p_{\gamma, 1}=0$ & $\hat{T}_{2}=0$ & $p_{\gamma, 2}=0$ & $\ldots$ \\
\hline$C\left[Y_{0}\right]$ & 0 & 0 & 0 & 0 & $\ldots$ \\
$C_{\theta}\left[Y_{0}\right]$ & $\frac{\hat{T}_{0}}{2 \sqrt{3 \pi}}$ & 0 & 0 & 0 & $\ldots$ \\
\hline$C\left[Y_{1}\right]$ & $-\frac{p_{\gamma 0}}{2 \sqrt{\pi}}$ & $\frac{\hat{T}_{0}}{\sqrt{\pi}}$ & 0 & 0 & $\ldots$ \\
$C_{\theta}\left[Y_{1}\right]$ & 0 & $*$ & $\frac{\hat{T}_{0}}{\sqrt{15 \pi}}$ & 0 & $\ldots$ \\
\hline$C\left[Y_{2}\right]$ & 0 & $\star$ & $-\frac{p_{\gamma 0}}{2 \sqrt{\pi}}$ & $\frac{3 \hat{T}_{0}}{\sqrt{4 \pi}}$ & $\ldots$ \\
$C_{\theta}\left[Y_{2}\right]$ & $\frac{\hat{T}_{0}}{\sqrt{15 \pi}}$ & $*$ & 0 & $*$ & $\ldots$ \\
\hline$\vdots$ & $\vdots$ & $\vdots$ & $\vdots$ & $\vdots$ & $\ddots$ \\
\hline \hline
\end{tabular}

One must also check if the polar constraints are gauge fixed by our conditions (4.19). To 
this end we compute

$$
\begin{aligned}
& \left\{\hat{T}_{n}, \hat{T} e^{-\hat{\gamma} / 2}\right\} \approx 0 \\
& \left\{p_{\hat{\gamma} n}, \hat{T} e^{-\hat{\gamma} / 2}\right\} \approx \frac{1}{2} \hat{T} e^{-\hat{\gamma} / 2} \sqrt{\frac{2 n+1}{4 \pi}} P_{n}(\cos \theta) .
\end{aligned}
$$

The last Poisson bracket is different from zero at the poles $(\theta=0, \pi)$ for all values of $n \in \mathbb{N}$. As we can see the only constraint that is not gauge-fixed by the conditions introduced above, as long as $p_{\gamma_{0}} \neq 0$ and $\hat{T}_{0} \neq 0$, is $C[1]$. This is different from the situation in the $\mathbb{T}^{2}$ case where we were left with two constraints instead of just one.

As we did before we can pullback everything to the phase space hypersurface defined by the gauge fixing conditions. The induced 2-form becomes

$$
\iota^{*} \omega=\mathrm{d} \hat{\gamma}_{0} \wedge \mathrm{d} p_{\hat{\gamma}_{0}}+\mathrm{d} \hat{T}_{0} \wedge \mathrm{d} p_{\hat{T}_{0}}+\sum_{i} \int_{\mathbb{S}^{2}}{ }^{(2)} e \delta \phi_{i} \wedge \delta p_{\phi_{i}}
$$

and the remaining constraint is

$$
\begin{aligned}
\mathcal{C} & :=-p_{\hat{\gamma}_{0}} p_{\hat{T}_{0}}+\hat{T}_{0}\left[4 \sqrt{\pi}\left(\log \frac{\hat{T}_{0}}{\sqrt{4 \pi}}-1\right)-\hat{\gamma}_{0}\right] \\
& +\frac{1}{2} \sum_{i} \int_{\mathbb{S}^{2}}{ }^{(2)} \mathrm{e}\left(\frac{\sqrt{4 \pi}}{\hat{T}_{0}} p_{\hat{\phi}_{i}}^{2}+\frac{\hat{T}_{0}}{\sqrt{4 \pi}} \hat{\phi}_{i}^{\prime 2} \sin ^{2} \theta\right) \approx 0 .
\end{aligned}
$$

The gauge transformations generated by this constraint in the variables $\hat{T}_{0}$ and $p_{\hat{\gamma}_{0}}$ are

$$
\begin{aligned}
& \left\{\hat{T}_{0}, \mathcal{C}\right\}=-p_{\hat{\gamma}_{0}}, \\
& \left\{p_{\hat{\gamma}_{0}}, \mathcal{C}\right\}=\hat{T}_{0},
\end{aligned}
$$

so if we parameterize the gauge orbits as before with $s \in(0, \pi)$ we find now $\hat{T}_{0}=p \sin s$ and $p_{\hat{\gamma}_{0}}=-p \cos s, p \neq 0$. In the spirit of the previous section we introduce now the following canonical transformation $\left((Q, P)\right.$ and $\left(T, p_{T}\right)$ denote canonically conjugate pairs)

$$
\begin{gathered}
\hat{T}_{0}=P \sin T, \quad p_{\hat{T}_{0}}=\frac{p_{T}}{P} \cos T-Q \sin T, \\
\hat{\gamma}_{0}=-Q \cos T-\frac{p_{T}}{P} \sin T, p_{\hat{\gamma}_{0}}=-P \cos T .
\end{gathered}
$$

In addition, as we did in the $\mathbb{T}^{3}$ case, it is possible to write the remaining constraint in a more pleasant form by performing a further canonical transformation (here, again, $(\tilde{Q}, \tilde{P})$ and $\left(\varphi_{i}, p_{\varphi_{i}}\right)$ are canonical pairs)

$$
\begin{array}{rlrl}
\tilde{Q} & :=P Q+\frac{1}{2} \sum_{i} \int_{\mathbb{S}^{2}}{ }^{(2)} \mathrm{e} p_{\hat{\phi}_{i}} \hat{\phi}_{i}, \quad \tilde{P}:=\log P, \\
\varphi_{i} & =(4 \pi)^{-1 / 4} \sqrt{P} \hat{\phi}_{i}, & p_{\varphi_{i}} & =(4 \pi)^{1 / 4} \frac{p_{\hat{\phi}_{i}}}{\sqrt{P}},
\end{array}
$$


giving

$$
\mathcal{C}=p_{T}+4 \sqrt{\pi} e^{\tilde{P}}\left(\log \frac{\sin T}{\sqrt{4 \pi}}+\tilde{P}-1\right) \sin T+\frac{1}{2} \sum_{i} \int_{\mathbb{S}^{2}}{ }^{(2)} \mathrm{e}\left(\frac{p_{\varphi_{i}}^{2}}{\sin T}+\varphi_{i}^{\prime 2} \sin T \sin ^{2} \theta\right) \approx 0 .
$$

It is now obvious the interpretation of the system as a non-autonomous Hamiltonian system $\left((0, \pi) \times \Gamma_{R}, \mathrm{~d} t, \omega_{R}, H_{R}\right)$, where $\Gamma_{R}$ denotes the reduced phase space coordinatized by the canonical pairs $\left(\tilde{Q}, \tilde{P} ; \varphi_{i}, p_{\varphi_{i}}\right)$, endowed with the standard (weakly) simplectic form (3.22). The dynamics is given by the time dependent Hamiltonian $H_{R}(t): \Gamma_{R} \rightarrow \mathbb{R}$

$$
H_{R}(t)=4 \sqrt{\pi} e^{\tilde{P}}\left(\log \frac{\sin t}{\sqrt{4 \pi}}+\tilde{P}-1\right) \sin t+\frac{1}{2} \sum_{i} \int_{\mathbb{S}^{2}}{ }^{(2)} \mathrm{e}\left(\frac{p_{\varphi_{i}}^{2}}{\sin t}+\varphi_{i}^{\prime 2} \sin t \sin ^{2} \theta\right),
$$

with the evolution vector field

$$
\begin{aligned}
E_{H_{R}}= & \frac{\partial}{\partial t}+4 \sqrt{\pi} e^{\tilde{P}}\left(\log \frac{\sin t}{\sqrt{4 \pi}}+\tilde{P}\right) \sin t \frac{\partial}{\partial \tilde{Q}} \\
& +\sum_{i} \int_{\mathbb{S}^{2}}{ }^{(2)} \mathrm{e}\left(\frac{p_{\varphi_{i}}}{\sin t} \frac{\delta}{\delta \varphi_{i}}+\left(\sin ^{2} \theta \varphi_{i}^{\prime}\right)^{\prime} \sin t \frac{\delta}{\delta p_{\varphi_{i}}}\right) .
\end{aligned}
$$

Several comments are in order at this point. First we can see now that the final description of our system is somewhat simpler that in the $\mathbb{T}^{3}$ case because we do not have any remaining constraints and the fields $\varphi_{1}$ and $\varphi_{2}$ are decoupled (at variance with the previous case). On the other hand we see now that the dynamics of the global modes, though easy to get in explicit form, is not as simple as the one found for the torus. Notice also that the Hamiltonian (4.24) is singular whenever $\sin t=0$. This means that if we pick the initial time $t_{0} \in(0, \pi)$ in order to write the Cauchy data we meet a past singularity at $t=0$ and a future singularity at $t=\pi$.

\section{V. $\mathbb{S}^{3}$ GOWDY MODELS COUPLED TO MASSLESS SCALARS}

Let us finally consider the case where the spatial slices ${ }^{(3)} \Sigma$ have the topology of a 3sphere $\mathbb{S}^{3}$, described as $\mathbb{S}^{3}=\left\{\left(z_{1}, z_{2}\right) \in \mathbb{C}^{2}:\left|z_{1}\right|^{2}+\left|z_{2}\right|^{2}=1\right\}$. Let us define the following action of $G^{(2)}$ on ${ }^{(3)} \Sigma$

$$
\left(g_{1}, g_{2}\right) \cdot\left(z_{1}, z_{2}\right)=\left(e^{i x_{1}}, e^{i x_{2}}\right) \cdot\left(z_{1}, z_{2}\right)=\left(e^{i x_{1}} z_{1}, e^{i x_{2}} z_{2}\right)
$$

The action of the two $U(1)$ subgroup factors is

$$
\begin{aligned}
& \left(e^{i x}, 1\right) \cdot\left(z_{1}, z_{2}\right)=\left(e^{i x} z_{1}, z_{2}\right) \\
& \left(1, e^{i x}\right) \cdot\left(z_{1}, z_{2}\right)=\left(z_{1}, e^{i x} z_{2}\right) .
\end{aligned}
$$


The corresponding tangent vectors at each point of $\mathbb{S}^{3}$, obtained by differentiating the previous expressions with respect to $x$ at $x=0$, are now

$$
\begin{aligned}
& \left(i z_{1}, 0\right) \\
& \left(0, i z_{2}\right) .
\end{aligned}
$$

As we can see they vanish at $z_{1}=0$ and $z_{2}=0$ (i.e. at the circumferences $\left(0, e^{i \xi}\right)$ and $\left(e^{i \sigma}, 0\right)$, $\xi, \sigma \in \mathbb{R}(\bmod 2 \pi))$. This fact poses now the question of how one can possibly use them to perform a Geroch reduction that requires us to have at least a non-vanishing Killing vector field. On some other respects they present no problems, in particular they are commuting fields. A useful parametrization of $\mathbb{S}^{3}$ is $z_{1}=e^{i \sigma} \sin (\theta / 2), z_{2}=e^{i \xi} \cos (\theta / 2)$ with $\theta \in[0, \pi]$, $\xi, \sigma \in \mathbb{R}(\bmod 2 \pi)$, with the commuting Killing fields $\sigma^{a}$ and $\xi^{a}$ given by $\sigma^{a}=(\partial / \partial \sigma)^{a}$ and $\xi^{a}=(\partial / \partial \xi)^{a}$. This allows us to view the three-sphere as a filled torus in which the points on the same parallel of the surface are identified (so that the surface itself can be viewed as a circle $\mathbb{S}^{1}$ ). This is helpful to perform the Geroch reduction.

The fact that the Killing vectors that we have chosen vanish alternatively in two different circles poses a problem as far as the Geroch reduction is concerned because to perform it one should use a non-vanishing vector. We will show now that the the fact that $\xi^{a}$ only vanishes in a one dimensional submanifold will effectively allow us to use them to carry out this reduction. To this end we start form an action in four dimensions defined on ${ }^{(4)} \mathcal{M}$, topologically $\mathbb{R} \times \mathbb{S}^{3}$, and remove the circle where the Killing vanishes from the integration region. As this is a zero-measure set the integral will not change. Of course one must take now into account the fact that the fields in the new integration region cannot be completely arbitrary but should be subject to some restrictions (regularity conditions) reflecting the fact that they should extend to the full ${ }^{(4)} \mathcal{M}$ in a smooth way.

Topologically the two dimensional manifold that appears in the action $(2.3)$ is ${ }^{(2)} \Sigma=D$, where $D$ denotes an open disk. The regularity conditions on the disk boundary are such that the fields (of any tensor type) behave in a "radial coordinate" $\theta$ exactly as an axially symmetric field would do in the axis. Eventually this will allow us to change the disk by a two sphere.

As in the previous cases we are going to use $\left(t^{a}, \theta^{a}, \sigma^{a}\right)$ as coordinate vector fields. We will write now $\theta^{a}=f \hat{\theta}^{a}, t^{a}=f\left(N n^{a}+N^{\theta} \hat{\theta}^{a}\right)$ with $N>0$ and $f>0$. The scalars $f, N$, and $N^{\theta}$ are supposed to be smooth fields on $\mathbb{R} \times D$ subject to some regularity conditions that will be specified later. Notice that we write now $f$ instead of $e^{\gamma} / 2$ as in previous cases because we want to allow $f$ to go to zero at the disk boundary. The same argument that we used for the two previous cases tells us now that

$$
\begin{aligned}
& N \mathcal{L}_{\theta} f+f \mathcal{L}_{\theta} N+N f^{2} n^{a} n^{b} \nabla_{a} \hat{\theta}_{b}=0, \\
& N^{\theta} \mathcal{L}_{\theta} f+f \mathcal{L}_{\theta} N^{\theta}-\mathcal{L}_{t} f+N f^{2} \hat{\theta}^{a} \hat{\theta}^{b} \nabla_{a} n_{b}=0, \\
& \hat{\theta}^{a} \sigma^{b} \nabla_{a} n_{b}=0 .
\end{aligned}
$$

The form of the 3-metric $g_{a b}$ is basically the same as in the other cases

$$
g_{a b}=f^{2}\left[\left(N^{\theta 2}-N^{2}\right)(\mathrm{d} t)_{a}(\mathrm{~d} t)_{b}+2 N^{\theta}(\mathrm{d} t)_{(a}(\mathrm{d} \theta)_{b)}+(\mathrm{d} \theta)_{a}(\mathrm{~d} \theta)_{b}\right]+\tau^{2}(\mathrm{~d} \sigma)_{a}(\mathrm{~d} \sigma)_{b}
$$

and the determinant is now given by $|g|=\tau^{2} N^{2} f^{4}$. Again $\left(N, N^{\theta}, \gamma, \phi_{i}\right)$, are constant on the orbits of the remaining Killing field $\sigma^{a}$ and hence they only depend on the coordinates 
$(t, \theta)$. Using the coordinates system $(t, \theta, \sigma, \xi)$ we write the original 4 -metric ${ }^{(4)} g_{a b}$ as

$$
\begin{aligned}
{ }^{(4)} g_{a b} & =\frac{f^{2}}{\lambda_{\xi}}\left[\left(N^{\theta 2}-N^{2}\right)(\mathrm{d} t)_{a}(\mathrm{~d} t)_{b}+2 N^{\theta}(\mathrm{d} t)_{(a}(\mathrm{d} \theta)_{b)}+(\mathrm{d} \theta)_{a}(\mathrm{~d} \theta)_{b}\right] \\
& +\frac{\tau^{2}}{\lambda_{\xi}}(\mathrm{d} \sigma)_{a}(\mathrm{~d} \sigma)_{b}+\lambda_{\xi}(\mathrm{d} \xi)_{a}(\mathrm{~d} \xi)_{b}
\end{aligned}
$$

We have to find out the regularity conditions satisfied by this metric. At $\theta=0$ the regularity conditions should be of the same type as the ones that we have already used in the $\mathbb{S}^{1} \times \mathbb{S}^{2}$ case. Here, however, we also have to impose regularity conditions when we approach the boundary of the filled torus that we obtained by removing the circle where the Killing used to perform the Geroch reduction vanishes. This can be formally achieved by changing $\theta$ for $\pi-\theta$. By doing this we find

$$
\begin{aligned}
& \frac{f^{2}}{\lambda_{\xi}}\left(N^{\theta 2}-N^{2}\right)=A(t, \cos \theta), \\
& \frac{f^{2}}{\lambda_{\xi}} N^{\theta}=B(t, \cos \theta) \sin \theta, \\
& \lambda_{\xi}=4 \cos ^{2}(\theta / 2)\left[F(t, \cos \theta)-G(t, \cos \theta) \cos ^{2}(\theta / 2)\right], \\
& \frac{f^{2}}{\lambda_{\xi}}=D(t, \cos \theta)+E(t, \cos \theta) \sin ^{2}(\theta / 2)= \\
& \frac{\tau^{2}}{\lambda_{\xi}}=4(t, \cos \theta)+G(t, \cos \theta) \cos ^{2}(\theta / 2),
\end{aligned}
$$

Here the functions $A, B, D, E, F$, and $G$ are analytic in their arguments. Notice that they are not independent because they are constrained to satisfy

$$
D(t, \cos \theta)+E(t, \cos \theta) \sin ^{2}(\theta / 2)=F(t, \cos \theta)+G(t, \cos \theta) \cos ^{2}(\theta / 2) .
$$

We have used the functions $\sin (\theta / 2)$ and $\cos (\theta / 2)$ because they alternatively vanish on the circles where the Killings themselves become zero and have the dependence of a regular scalar function in terms of the "radial" coordinates $\theta$ or $\pi-\theta$ on the circles where they do not vanish. The cosine dependence of the other functions is dictated by regularity at the two circles. This is very important because we will be able to write down our model in terms of them and, having $\cos \theta$ as their argument they can be interpreted as functions on $\mathbb{S}^{2}$ as in the $\mathbb{S}^{1} \times \mathbb{S}^{2}$ case. The conditions that the fields must satisfy (dropping the $t$-dependence) 
are

$$
\begin{aligned}
& \lambda_{\xi}=e^{\hat{\phi}_{1}(\cos \theta)} \cos ^{2}(\theta / 2) \\
& \phi_{2}=\hat{\phi}_{2}(\cos \theta) \\
& f=\cos (\theta / 2) e^{\hat{\gamma}(\cos \theta) / 2} \\
& N^{\theta}=\hat{N}^{\theta}(\cos \theta) \sin \theta \\
& N=\hat{N}(\cos \theta) \\
& \tau=\hat{T}(\cos \theta) \sin \theta \\
& \hat{T}^{2} e^{-\hat{\gamma}}=\frac{D(\cos \theta)-E(\cos \theta) \sin ^{2}(\theta / 2)}{D(\cos \theta)+E(\cos \theta) \sin ^{2}(\theta / 2)} \\
& e^{2 \hat{\phi}_{1}-\hat{\gamma}}=4 \frac{F(\cos \theta)-G(\cos \theta) \cos ^{2}(\theta / 2)}{F(\cos \theta)+G(\cos \theta) \cos ^{2}(\theta / 2)},
\end{aligned}
$$

where we have used $\sin \theta=2 \sin (\theta / 2) \cos (\theta / 2)$. Here, as in the $\mathbb{S}^{1} \times \mathbb{S}^{2}$ case, we have that $\hat{\phi}_{i}, \hat{\gamma}, \hat{N}^{\theta}, \hat{N}, \hat{T}:[-1,1] \rightarrow \mathbb{R}(\hat{N}>0)$. They can be written as functions of $A, B, D, E, F$, and $G$. They must be differentiable in $(-1,1)$, and their right and left derivatives at \pm 1 must be defined (equivalently they must be $\mathcal{C}^{\infty}$ in $(-1,1)$ with bounded derivative). Conditions (5.17,5.18) imply that

$$
\hat{T}(+1) e^{-\hat{\gamma}(+1) / 2}=1 \quad \text { and } \quad e^{2 \hat{\phi}_{1}(-1)-\hat{\gamma}(-1)}=4
$$

These are the polar constraints for the $\mathbb{S}^{3}$ topology. This is slightly different from previous examples because now these conditions involve different pairs of objets at the two poles of $\mathbb{S}^{2}$.

Our starting point is now the action

$$
{ }^{(3)} S\left(g_{a b}, \phi_{i}\right)=\frac{1}{16 \pi G_{3}} \int_{t_{0}}^{t_{1}} \mathrm{~d} t \int_{\mathbb{S}^{2}}{ }^{(2)} \mathrm{e}|g|^{1 / 2}\left({ }^{(2)} R+K_{a b} K^{a b}-K^{2}-\frac{1}{2} g^{a b} \sum_{i}\left(\mathrm{~d} \phi_{i}\right)_{a}\left(\mathrm{~d} \phi_{i}\right)_{b}\right) \text {, }
$$

with $\phi_{1}=\log \lambda_{\xi}=\hat{\phi}_{1}+\log \cos ^{2}(\theta / 2)$. Notice that we have changed the integration region to $\mathbb{S}^{2}$ because, as we will see, it can be written in terms of the hat-fields that are smoothly extended to $\mathbb{S}^{2}$.

As in the case of the three-handle we choose the fiducial volume element ${ }^{(2)} \mathrm{e}$ to be compatible with the auxiliary round metric on the 2 -sphere $\mathbb{S}^{2}$, i.e. ${ }^{(2)} \mathrm{e}=\sin \theta \mathrm{d} \theta \wedge \mathrm{d} \sigma$, with 
${ }^{(2)} \mathrm{e}_{a b} \theta^{a} \sigma^{b}=N f^{2} \tau /|g|^{1 / 2}=\sin \theta$. In terms of the fields $\left(\hat{N}, \hat{N}^{\theta}, \hat{\gamma}, \hat{T}, \hat{\phi}_{i}\right)$ the action becomes

$$
\begin{aligned}
& { }^{(3)} S\left(\hat{N}, \hat{N}^{\theta}, \hat{\gamma}, \hat{T}, \hat{\phi}_{i}\right)= \\
& =\frac{1}{16 \pi G_{3}} \int_{t_{0}}^{t_{1}} \mathrm{~d} t \int_{\mathbb{S}^{2}}^{\left({ }^{2}\right)} \mathrm{e}\left(\hat{N}\left[\left(\hat{\gamma}^{\prime} \hat{T}^{\prime}-2 \hat{T}^{\prime \prime}\right) \sin ^{2} \theta+\left(5 \hat{T}^{\prime}-\hat{\gamma}^{\prime} \hat{T}\right) \cos \theta+\hat{T}^{\prime}+\frac{3}{2} \hat{T}\right]\right. \\
& \quad+\frac{1}{\hat{N}}\left[\hat{N}^{\theta} \hat{T} \cos \theta-\hat{\hat{T}}-\hat{N}^{\theta} \hat{T}^{\prime} \sin ^{2} \theta\right]\left[\hat{\hat{\gamma}}+\left(2 \hat{N}^{\theta \prime}+\hat{N}^{\theta} \hat{\gamma}^{\prime}\right) \sin ^{2} \theta+(1-3 \cos \theta) \hat{N}^{\theta}\right] \\
& \quad+\frac{\hat{T}}{2 \hat{N}} \sum_{i}\left[\dot{\hat{\phi}}_{i}^{2}+2 \hat{N}^{\theta} \dot{\hat{\phi}}_{i} \hat{\phi}_{i}^{\prime} \sin ^{2} \theta+\left(\hat{N}^{\theta 2} \sin ^{2} \theta-\hat{N}^{2}\right) \hat{\phi}_{i}^{\prime 2} \sin ^{2} \theta\right] \\
& \left.\quad+\frac{\hat{T}}{2 \hat{N}}\left[2(1-\cos \theta)\left(\hat{N}^{\theta} \dot{\hat{\phi}}_{1}+\left(\hat{N}^{\theta 2} \sin ^{2} \theta-\hat{N}^{2}\right) \hat{\phi}_{1}^{\prime}\right)+(1-\cos \theta)^{2} \hat{N}^{\theta 2}\right]\right) .
\end{aligned}
$$

It is important to remark at this point that the action is the integral of a smooth function on the sphere. We arrive at this result after several non-trivial cancelations of terms that would diverge at the poles. This reflects the fact that indeed, by removing the circle where the Killing vector field used in the Geroch reduction vanishes, we arrive at a consistent description of the model. It is also worthwhile pointing out that the structure of the action is very similar to the one found in the $\mathbb{S}^{1} \times \mathbb{S}^{2}$ case but not exactly the same, in fact we will see later that the differences are important to guarantee, for example, the stability of the polar constraints in this case.

The Hamiltonian of the system can be readily obtained. As in previous cases it can be written as a sum of constraints $H=C[\hat{N}]+C_{\theta}\left[\hat{N}^{\theta}\right]$ with

$$
\begin{gathered}
C[\hat{N}]=\int_{\mathbb{S}^{2}}{ }^{(2)} \mathrm{e} \hat{N} C \\
=\int_{\mathbb{S}^{2}}^{(2)} \mathrm{e} \hat{N}\left(-16 \pi G_{3} p_{\hat{\gamma}} p_{\hat{T}}+\frac{1}{16 \pi G_{3}}\left[\left(2 \hat{T}^{\prime \prime}-\hat{\gamma}^{\prime} \hat{T}^{\prime}\right) \sin ^{2} \theta+\left(\hat{\gamma}^{\prime} \hat{T}-5 \hat{T}^{\prime}\right) \cos \theta-\frac{3}{2} \hat{T}-\hat{T}^{\prime}\right]\right. \\
\left.\quad+\frac{1}{2} \sum_{i}\left(\frac{16 \pi G_{3} p_{\hat{\phi}_{i}}^{2}}{\hat{T}}+\frac{\hat{T}}{16 \pi G_{3}} \hat{\phi}_{i}^{\prime 2} \sin ^{2} \theta\right)+(1-\cos \theta) \frac{\hat{T}}{16 \pi G_{3}} \hat{\phi}_{1}^{\prime}\right) \\
C_{\theta}\left[\hat{N}^{\theta}\right]=\int_{\mathbb{S}^{2}}{ }^{(2)} \mathrm{e} \hat{N}^{\theta} C_{\theta} \\
=\int_{\mathbb{S}^{2}}^{(2)} \hat{\mathrm{N}}^{\theta}\left(\left[2 p_{\hat{\gamma}}^{\prime}-\hat{\gamma}^{\prime} p_{\hat{\gamma}}-\hat{T}^{\prime} p_{\hat{T}}-\sum_{i} \hat{\phi}_{i}^{\prime} p_{\hat{\phi}_{i}}\right] \sin ^{2} \theta+\left[\hat{T} p_{\hat{T}}-p_{\hat{\gamma}}+p_{\hat{\phi}_{1}}\right] \cos \theta-p_{\hat{\gamma}}-p_{\hat{\phi}_{1}}\right) .
\end{gathered}
$$

The two previous expressions, together with the conditions at the poles $\hat{T}(+1) e^{-\hat{\gamma}(+1) / 2}=1$ and $e^{2 \hat{\phi}_{1}(-1)-\hat{\gamma}(-1)}=4$, define the constraints of the system. As before, the polar constraints are necessary conditions to guarantee the differentiability of the (weighted) constraints $C\left[N_{g}\right]$ 
and $C_{\theta}\left[N_{g}^{\theta}\right]$. The gauge transformations defined by $C\left[\hat{N}_{g}\right]$ and $C_{\theta}\left[\hat{N}_{g}^{\theta}\right]$ are $^{11}$

$$
\begin{aligned}
\left\{\hat{\gamma}, C\left[\hat{N}_{g}\right]\right\}= & -\hat{N}_{g} p_{\hat{T}}, \\
\left\{\hat{T}, C\left[\hat{N}_{g}\right]\right\}= & -\hat{N}_{g} p_{\hat{\gamma}} \\
\left\{\hat{\phi}_{i}, C\left[\hat{N}_{g}\right]\right\}= & \hat{N}_{g} \frac{p_{\hat{\phi}_{i}}}{\hat{T}} \\
\left\{p_{\hat{\gamma}}, C\left[\hat{N}_{g}\right]\right\}= & \hat{N}_{g}^{\prime}\left(\hat{T} \cos \theta-\hat{T}^{\prime} \sin ^{2} \theta\right)+\hat{N}_{g}\left(3 \hat{T}^{\prime} \cos \theta+\hat{T}-\hat{T}^{\prime \prime} \sin ^{2} \theta\right), \\
\left\{p_{\hat{T}}, C\left[\hat{N}_{g}\right]\right\}= & \hat{N}_{g}\left[\frac{1}{2}-\hat{\phi}_{1}^{\prime}+\left(\hat{\gamma}^{\prime}+\hat{\phi}_{1}^{\prime}\right) \cos \theta-\hat{\gamma}^{\prime \prime} \sin ^{2} \theta\right]+\hat{N}_{g}^{\prime}\left(3 \cos \theta-1-\hat{\gamma}^{\prime} \sin ^{2} \theta\right) \\
& -2 \hat{N}_{g}^{\prime \prime} \sin ^{2} \theta+\frac{\hat{N}_{g}}{2} \sum_{i}\left(\frac{p_{\hat{\phi}_{i}}^{2}}{\hat{T}^{2}}-\sin ^{2} \theta \hat{\phi}_{i}^{\prime 2}\right), \\
\left\{p_{\hat{\phi}_{1}}, C\left[\hat{N}_{g}\right]\right\}= & {\left[\hat{N}_{g} \hat{T}\left(\hat{\phi}_{2}^{\prime} \sin ^{2} \theta+1-\cos \theta\right)\right]^{\prime}, } \\
\left\{p_{\hat{\phi}_{2}}, C\left[\hat{N}_{g}\right]\right\}= & \left(\hat{N}_{g} \hat{T} \hat{\phi}_{2}^{\prime} \sin ^{2} \theta\right)^{\prime},
\end{aligned}
$$

and

$$
\begin{aligned}
& \left\{\hat{\gamma}, C_{\theta}\left[\hat{N}_{g}^{\theta}\right]\right\}=-2 \hat{N}_{g}^{\theta \prime} \sin ^{2} \theta+\hat{N}_{g}^{\theta}\left(3 \cos \theta-\hat{\gamma}^{\prime} \sin ^{2} \theta-1\right), \\
& \left\{\hat{T}, C_{\theta}\left[\hat{N}_{g}^{\theta}\right]\right\}=\hat{N}_{g}^{\theta}\left(\hat{T} \cos \theta-\hat{T}^{\prime} \sin ^{2} \theta\right) \\
& \left\{\hat{\phi}_{1}, C_{\theta}\left[\hat{N}_{g}^{\theta}\right]\right\}=\hat{N}_{g}^{\theta}\left(\cos \theta-1-\hat{\phi}_{2}^{\prime} \sin ^{2} \theta\right) \\
& \left\{\hat{\phi}_{2}, C_{\theta}\left[\hat{N}_{g}^{\theta}\right]\right\}=-\hat{N}_{g}^{\theta} \hat{\phi}_{2}^{\prime} \sin ^{2} \theta \\
& \left\{p_{\hat{\gamma}}, C_{\theta}\left[\hat{N}_{g}^{\theta}\right]\right\}=-\left(\hat{N}_{g}^{\theta} p_{\hat{\gamma}} \sin ^{2} \theta\right)^{\prime} \\
& \left\{p_{\hat{T}}, C_{\theta}\left[\hat{N}_{g}^{\theta}\right]\right\}=\hat{N}_{g}^{\theta}\left(p_{\hat{T}} \cos \theta-p_{\hat{T}^{\prime}}^{\prime} \sin ^{2} \theta\right)-\hat{N}_{g}^{\theta \prime} p_{\hat{T}} \sin ^{2} \theta \\
& \left\{p_{\hat{\phi}_{i}}, C_{\theta}\left[\hat{N}_{g}^{\theta}\right]\right\}=-\left(\hat{N}_{g}^{\theta} p_{\hat{\phi}_{i}} \sin ^{2} \theta\right)^{\prime}
\end{aligned}
$$

The Poisson brackets of these constraints give exactly the same result that we obtained for the $\mathbb{S}^{1} \times \mathbb{S}^{2}$ topology and, hence, define a fist class constrained surface $\Gamma_{c} \subset \Gamma$. Here $(\Gamma, \omega)$ denotes the canonical phase space of the system, coordinatized by the canonical pairs $\left(\hat{\gamma}, p_{\hat{\gamma}} ; \hat{T}, p_{\hat{T}} ; \hat{\phi}_{i}, p_{\hat{\phi}_{i}}\right)$, and endowed with the standard (weakly) symplectic form (4.16). We must check now the stability of the polar constraints. We do this by computing

$$
\begin{aligned}
& \left\{\hat{T} e^{-\hat{\gamma} / 2}, C\left[\hat{N}_{g}\right]\right\}=\frac{1}{2} \hat{N}_{g} e^{-\hat{\gamma} / 2}\left(\hat{T} p_{\hat{T}}-2 p_{\hat{\gamma}}\right), \\
& \left\{\hat{T} e^{-\hat{\gamma} / 2}, C_{\theta}\left[\hat{N}_{g}^{\theta}\right]\right\}=e^{-\hat{\gamma} / 2}\left(\frac{1}{2} \hat{N}_{g}^{\theta} \hat{T}(1-\cos \theta)+\left(\hat{N}_{g}^{\theta \prime} \hat{T}-\hat{N}_{g}^{\theta} \hat{T}^{\prime}+\frac{1}{2} \hat{N}_{g}^{\theta} \hat{T} \hat{\gamma}^{\prime}\right) \sin ^{2} \theta\right), \\
& \left\{e^{2 \hat{\phi}_{1}-\hat{\gamma}}, C\left[\hat{N}_{g}\right]\right\}=\frac{\hat{N}^{g}}{\hat{T}} e^{2 \hat{\phi}_{1}-\hat{\gamma}}\left(2 p_{\hat{\phi}_{1}}+\hat{T} p_{\hat{T}}\right), \\
& \left\{e^{2 \hat{\phi}_{1}-\hat{\gamma}}, C_{\theta}\left[\hat{N}_{g}^{\theta}\right]\right\}=e^{2 \hat{\phi}_{1}-\hat{\gamma}}\left(-\hat{N}_{g}^{\theta}(1+\cos \theta)+\left(2 \hat{N}_{g}^{\theta \prime}-2 \hat{N}_{g}^{\theta} \hat{\phi}_{2}^{\prime}+\hat{N}_{g}^{\theta} \hat{\gamma}^{\prime}\right) \sin ^{2} \theta\right) .
\end{aligned}
$$

${ }^{11}$ Again we take $16 \pi G_{3}=1$. 
The constraint (5.21) at the poles $\theta=0, \pi$ gives respectively, $\hat{T}(+1) p_{\hat{T}}(+1)-2 p_{\hat{\gamma}}(+1)=0$, and $\hat{T}(-1) p_{\hat{T}}(-1)+2 p_{\hat{\phi}_{1}}(-1)=0$. These guarantee that the Poisson bracket (5.22) , vanishes at $\theta=0$ and (5.24) vanishes at $\theta=\pi$. The vanishing of (5.23) at $\theta=0$ is due to the presence of the factors $1-\cos \theta$ and $\sin ^{2} \theta$ and, finally, (5.25) is zero at $\theta=\pi$ due to the factors $1+\cos \theta$ and $\sin ^{2} \theta$. As in the $\mathbb{S}^{1} \times \mathbb{S}^{2}$ we conclude that there are no secondary constraints coming from the

stability of these polar constraints.

The deparameterization in this case follows closely the one for $\mathbb{S}^{1} \times \mathbb{S}^{2}$. The same gauge fixing conditions work in our case now. The only new element now is checking if the polar constraints are gauge fixed or not and this only requires the computation of

$$
\left\{p_{\hat{\gamma} n}, e^{2 \hat{\phi}_{1}-\hat{\gamma}}\right\}=e^{2 \hat{\phi}_{1}-\hat{\gamma}} \sqrt{\frac{2 n+1}{4 \pi}} P_{n}(\cos \theta)
$$

which is different from zero at the poles. As we see the situation now is completely analogous to the previous case. The pull-back of the symplectic form to the phase space hypersurface defined by the gauge fixing conditions is given again by (4.20). We are left only with the constraint

$$
\begin{aligned}
\mathcal{C} & :=-p_{\hat{\gamma}_{0}} p_{\hat{T}_{0}}+\hat{T}_{0}\left(\sqrt{4 \pi} \log \frac{\hat{T}_{0}}{\sqrt{4 \pi}}-\hat{\gamma}_{0}-\sqrt{\pi}(2 \log 2+3)+\hat{\phi}_{1_{0}}\right) \\
& +\frac{1}{2} \sum_{i} \int_{\mathbb{S}^{2}}{ }^{(2)} \mathrm{e}\left(\frac{\sqrt{4 \pi} p_{\hat{\phi}_{i}}^{2}}{\hat{T}_{0}}+\frac{\hat{T}_{0}}{\sqrt{4 \pi}} \hat{\phi}_{i}^{\prime 2}\right) \approx 0 .
\end{aligned}
$$

The gauge transformations generated by this constraint on the variables $\hat{T}_{0}$ and $p_{\hat{\gamma} 0}$ are the same as for the three-handle and, hence, we can use the canonical transformations introduced at the end of the previous section to rewrite (5.26) as

$$
\begin{aligned}
p_{T} & +(4 \pi)^{1 / 4} e^{\tilde{P} / 2} \hat{\varphi}_{1_{0}} \sin T+2 \sqrt{\pi} e^{\tilde{P}}\left(\log \frac{\sin T}{\sqrt{4 \pi}}+\tilde{P}-\log 2-\frac{3}{2}\right) \sin T \\
& +\frac{1}{2} \sum_{i} \int_{\mathbb{S}^{2}}{ }^{(2)} \mathrm{e}\left(\frac{p_{\hat{\varphi}_{i}}^{2}}{\sin T}+\hat{\varphi}_{i}^{\prime 2} \sin T \sin ^{2} \theta\right) \approx 0 .
\end{aligned}
$$

The description of the system by a time-dependent Hamiltonian is now straightforward. It is interesting at this point to compare the dynamics of this model and the $\mathbb{S}^{1} \times \mathbb{S}^{2}$ one. First of all we see that the global mode have a different behavior now, in particular couples to $\varphi_{1_{0}}$ through the term $e^{\tilde{P} / 2} \hat{\varphi}_{1_{0}} \sin T$ in (5.27). As we see the gravitational and matter modes cease to play a symmetric role in this particular description, at variance with the other topologies. However, by writing the regularity condition (5.11) with an extra $\hat{T}$ (as will be justified in the next section) it is possible to restore the symmetry between the gravitational and matter scalars in a straightforward way.

As in the previous cases, it is possible to interpret the system as a non-autonomous Hamiltonian system $\left((0, \pi) \times \Gamma_{R}, \mathrm{~d} t, \omega_{R}, H_{R}\right)$, where $\Gamma_{R}$ denotes the reduced phase space coordinatized by the canonical pairs $\left(\tilde{Q}, \tilde{P} ; \varphi_{i}, p_{\varphi_{i}}\right)$, endowed with the standard (weakly) simplectic form (3.22). The dynamics is given by the time dependent Hamiltonian $H_{R}(t)$ : 
$\Gamma_{R} \rightarrow \mathbb{R}$

$$
\begin{aligned}
H_{R}(t) & =(4 \pi)^{1 / 4} e^{\tilde{P} / 2} \varphi_{1_{0}} \sin t+2 \sqrt{\pi} e^{\tilde{P}}\left(\log \frac{\sin t}{\sqrt{4 \pi}}+\tilde{P}-\log 2-\frac{3}{2}\right) \sin t \\
& +\frac{1}{2} \sum_{i} \int_{\mathbb{S}^{2}}{ }^{(2)} \mathrm{e}\left(\frac{p_{\varphi_{i}}^{2}}{\sin t}+\varphi_{i}^{\prime 2} \sin t \sin ^{2} \theta\right)
\end{aligned}
$$

with the evolution vector field given by

$$
\begin{aligned}
E_{H_{R}} & =\frac{\partial}{\partial t}+\left[(4 \pi)^{1 / 4} e^{\tilde{P} / 2} \varphi_{1_{0}} \sin t+2 \sqrt{\pi} e^{\tilde{P}} \sin t\left(\log \frac{\sin t}{\sqrt{4 \pi}}+\tilde{P}-\log 2-\frac{1}{2}\right)\right] \frac{\partial}{\partial \tilde{Q}} \\
& -(4 \pi)^{1 / 4} e^{\tilde{P} / 2} \sin t \frac{\partial}{\partial p_{\varphi_{1_{0}}}}+\sum_{i} \int_{\mathbb{S}^{2}}{ }^{(2)} \mathrm{e}\left(\frac{p_{\varphi_{i}}}{\sin t} \frac{\delta}{\delta \varphi_{i}}+\left(\sin ^{2} \theta \varphi_{i}^{\prime}\right)^{\prime} \sin t \frac{\delta}{\delta p_{\varphi_{i}}}\right) .
\end{aligned}
$$

The singularities in this case show up in the same way as for the $\mathbb{S}^{1} \times \mathbb{S}^{2}$ topology.

\section{GOWDY MODELS AS SCALAR FIELD THEORIES IN 2+1 CURVED BACKGROUND}

The purpose of this section is to reinterpret the reduced models presented in the previous sections as certain simple massless scalar field theories in conformally stationary backgrounds. We will show how the metrics obtained after the specific gauge fixing and deparameterization used in the previous sections can be employed to reinterpret the meaning (and solution) of the field equations for each topology. This will allow us to use well-known techniques of quantum field theory in curved backgrounds to quantize these systems [30].

Let us start by giving a simple way to solve equations (2.2)

$$
\begin{aligned}
& R_{a b}=\frac{1}{2} \sum_{i}\left(\mathrm{~d} \phi_{i}\right)_{a}\left(\mathrm{~d} \phi_{i}\right)_{b}, \\
& g^{a b} \nabla_{a} \nabla_{b} \phi_{i}=0 \\
& \mathcal{L}_{\sigma} \phi_{i}=0 .
\end{aligned}
$$

If a specific solution $\left(\stackrel{\circ}{g}_{a b}, \stackrel{\circ}{\phi}_{1}, \dot{\phi}_{2}\right)$ is known it is possible to decouple (6.1) and (6.2, 6.3) because, when (6.3) is satisfied, we have the equivalence

$$
g^{a b} \nabla_{a} \nabla_{b} \phi_{i}=0 \Leftrightarrow \stackrel{\circ}{g}^{a b} \stackrel{\circ}{\nabla}_{a} \stackrel{\circ}{\nabla}_{b} \phi_{i}=0 .
$$

The idea is then to solve the last equation in the background $\stackrel{\circ}{g}_{a b}$ and then equation (6.1) just gives integrability conditions allowing us to recover $g_{a b}$. We will discuss next the specific form of $\stackrel{\circ}{g}_{a b}$ for each of the spatial topologies considered in the paper.

\section{- Background metric for $\mathbb{T}^{3}$}

In this case the form of the metric $g_{a b}$ found after the deparameterization is

$$
g_{a b}=e^{\gamma}\left(-(\mathrm{d} t)_{a}(\mathrm{~d} t)_{b}+(\mathrm{d} \theta)_{a}(\mathrm{~d} \theta)_{b}\right)+\frac{P^{2} t^{2}}{2 \pi}(\mathrm{d} \sigma)_{a}(\mathrm{~d} \sigma)_{b}
$$


defined on $(0, \infty) \times \mathbb{T}^{2}$. A possible (non unique) choice for $\left(\stackrel{\circ}{g}_{a b}, \stackrel{\circ}{\phi}_{1}, \stackrel{\circ}{\phi}_{2}\right)$ is

$$
\begin{aligned}
\stackrel{\circ}{g}_{a b} & =t^{2}\left(-(\mathrm{d} t)_{a}(\mathrm{~d} t)_{a}+(\mathrm{d} \theta)_{a}(\mathrm{~d} \theta)_{b}+(\mathrm{d} \sigma)_{a}(\mathrm{~d} \sigma)_{b}\right) \\
\dot{\phi}_{1} & =\log t \\
\dot{\phi}_{2} & =0
\end{aligned}
$$

where it is important to notice that even though $\stackrel{\circ}{g}_{a b}$ is not stationary it is conformal to a (flat) stationary metric on $(0, \infty) \times \mathbb{T}^{2}$.

\section{- Background metric for $\mathbb{S}^{1} \times \mathbb{S}^{2}$}

After deparameterization we get now

$$
g_{a b}=e^{\gamma}\left(-(\mathrm{d} t)_{a}(\mathrm{~d} t)_{b}+(\mathrm{d} \theta)_{a}(\mathrm{~d} \theta)_{b}\right)+\frac{P^{2}}{4 \pi} \sin ^{2} t \sin ^{2} \theta(\mathrm{d} \sigma)_{a}(\mathrm{~d} \sigma)_{b}
$$

defined on $(0, \pi) \times \mathbb{S}^{2}$. In this case a convenient choice for $\left(\stackrel{\circ}{g}_{a b}, \stackrel{\circ}{1}_{1}, \stackrel{\circ}{\phi}_{2}\right)$ is

$$
\begin{aligned}
\stackrel{\circ}{g}_{a b} & =\sin ^{2} t\left(-(\mathrm{d} t)_{a}(\mathrm{~d} t)_{a}+(\mathrm{d} \theta)_{a}(\mathrm{~d} \theta)_{b}+\sin ^{2} \theta(\mathrm{d} \sigma)_{a}(\mathrm{~d} \sigma)_{b}\right) \\
\stackrel{\circ}{\phi}_{1} & =\log \sin (t / 2)-\log \cos (t / 2) \\
\dot{\circ}_{2} & =0 .
\end{aligned}
$$

Again this metric is not stationary but it is equal to a time dependent conformal factor times the Einstein static metric on $(0, \pi) \times \mathbb{S}^{2}$.

\section{- Background metric for $\mathbb{S}^{3}$}

Finally we have now

$$
g_{a b}=\cos ^{2}(\theta / 2) e^{\gamma}\left(-(\mathrm{d} t)_{a}(\mathrm{~d} t)_{b}+(\mathrm{d} \theta)_{a}(\mathrm{~d} \theta)_{b}\right)+\frac{P^{2}}{4 \pi} \sin ^{2} t \sin ^{2} \theta(\mathrm{d} \sigma)_{a}(\mathrm{~d} \sigma)_{b}
$$

defined on $(0, \pi) \times D$ where $D$ denotes the open disk introduced in the previous section. In this case, a possible choice of $\left(\stackrel{\circ}{g}_{a b}, \dot{\phi}_{1}, \dot{\phi}_{2}\right)$ is

$$
\begin{aligned}
\stackrel{\circ}{g}_{a b} & =\cos ^{2}(\theta / 2) e^{\gamma}\left(-(\mathrm{d} t)_{a}(\mathrm{~d} t)_{b}+(\mathrm{d} \theta)_{a}(\mathrm{~d} \theta)_{b}\right)+\sin ^{2} t \sin ^{2} \theta(\mathrm{d} \sigma)_{a}(\mathrm{~d} \sigma)_{b} \\
\dot{\phi}_{1} & =\cos \theta \cos t \log (\tan (t / 2))+\cos \theta+\log \left(\cos ^{2}(\theta / 2)\right)+\log (2 \sin t) \\
\dot{\phi}_{2} & =0
\end{aligned}
$$

where

$$
\begin{aligned}
\dot{\gamma} & =\frac{\sin ^{2} \theta}{4}\left(\sin ^{2} t \log ^{2}(\tan t / 2)-2 \cos t \log (\tan t / 2)-1\right)+\log \left(\sin ^{2} t\right) \\
& -\cos t \log (\tan (t / 2))+\cos \theta \cos t \log (\tan (t / 2))+\cos \theta-1 .
\end{aligned}
$$

It is important to realize that the concrete functional form of $\dot{\gamma}$ is irrelevant because, 
whenever $\mathcal{L}_{\sigma} \phi_{i}=0$, we have the following equivalence in $(0, \pi) \times\left(\mathbb{S}^{2}-\{\theta=\pi\}\right)$

$$
\stackrel{\circ}{g}^{a b} \stackrel{\circ}{\nabla}_{a} \stackrel{\circ}{\nabla}_{b} \phi_{i}=0 \Leftrightarrow \breve{g}^{a b} \breve{\nabla}_{a} \breve{\nabla}_{b} \phi_{i}=0
$$

with

$$
\breve{g}_{a b}=\sin ^{2} t\left(-(\mathrm{d} t)_{a}(\mathrm{~d} t)_{b}+(\mathrm{d} \theta)_{a}(\mathrm{~d} \theta)_{b}+\sin ^{2} \theta(\mathrm{d} \sigma)_{a}(\mathrm{~d} \sigma)_{b}\right) .
$$

Notice that the metric $\breve{g}_{a b}$ is the one that we found for $(0, \pi) \times \mathbb{S}^{2}$ restricted to the manifold $(0, \pi) \times D$ obtained by removing a point from the sphere.

It is important to point out that $\phi_{1}$ cannot be extended to the boundary of the disk, parameterized as $\theta=\pi$, because (5.11) forces $\phi_{1}$ to behave as $\log \left(\cos ^{2}(\theta / 2)\right)$ for $\theta \rightarrow \pi$. However if we split $\phi_{1}$ as $\phi_{1}=\phi_{1}^{\text {sing }}+\phi_{1}^{\text {reg }}$ with $\phi_{1}^{\text {sing }}=\log \left(\cos ^{2}(\theta / 2)\right)+\log (2 \sin t)$, satisfying

$$
\breve{g}^{a b} \breve{\nabla}_{a} \breve{\nabla}_{b} \phi_{1}^{\text {sing }}=0,
$$

we guarantee that the degrees of freedom contained in $\phi_{1}^{\text {reg }}$ still satisfy $\breve{g}^{a b} \breve{\nabla}_{a} \breve{\nabla}_{b} \phi_{1}^{\text {reg }}=0$ (just the same equation as the matter field $\phi_{2}$ ) and can be extended to $(0, \pi) \times \mathbb{S}^{2}$. Notice that the role of the scalar fields $\phi_{1}^{\text {reg }}$ and $\phi_{2}$, both well behaved on $(0, \pi) \times \mathbb{S}^{2}$, is symmetric just as in the description of the previous topologies.

It is important to notice that the scalar field dynamics generated by the time dependent Hamiltonians that we have obtained in the previous sections corresponds exactly to the one defined by the Klein-Gordon equations on the backgrounds given by $\stackrel{\circ}{g}_{a b}$.

To end this section we want to point out that there are certain obstructions to the unitary implementation of quantum dynamics for these systems. Specifically, it can be shown that it is impossible to find a Fock space representation in which time evolution is unitarily implementable [10, 13]. The solution to this problem for the torus case relies on certain field redefinitions involving functions of time [7, 8]. These can be understood in the present scheme as coming from the time dependent conformal factors appearing in $\stackrel{\circ}{a b}_{a b}$ (or $\breve{g}_{a b}$ ). As we will show in a forthcoming paper, the solution to the unitarity problem for the topologies considered here relies on field redefinitions involving precisely the conformal factors shown above. Indeed, by performing a redefinition of the scalar fields at the Lagrangian level, such that the conformal factor relating both metrics is traded by a time-dependent potential term, we expect to find unitary dynamics if this potential is well behaved. In these cases the background metric corresponds to a simple, fixed stationary background.

\section{CONCLUSIONS AND COMMENTS}

We have studied in this paper the Hamiltonian formalism for the compact, linearly polarized Gowdy models coupled to massless scalar fields. The purpose of the analysis is to have a Hamiltonian formulation of the models that can be a starting point for their quantization either à la Dirac or by gauge fixing and deparameterization. The results for the $\mathbb{T}^{3}$ topology reproduce the known ones for the gravitational sector and show that the interaction of the matter fields occur though the constraints left over by the deparameterization of the system. In the other two cases the coupling of matter and gravity degrees of freedom can only be seen when the four metric is reconstructed. 
The description of the $\mathbb{S}^{1} \times \mathbb{S}^{2}$ and $\mathbb{S}^{3}$ models requires a careful discussion of the regularity conditions that the metric must satisfy in the symmetry axis left over after the Geroch reduction performed to describe the systems in $2+1$ dimensions. These regularity conditions are responsible for the appearance of the so called polar constraints. These can be shown to be first class and are necessary conditions to guarantee the differentiability of the other constraints present in the models. Of course they must be taken into account in a Dirac quantization of the Gowdy models corresponding to these topologies.

An interesting feature of both the $\mathbb{S}^{1} \times \mathbb{S}^{2}$ and $\mathbb{S}^{3}$ cases is the fact that after the deparameterization introduced in the paper (which is a straightforward generalization of the ones used in the literature for the familiar $\mathbb{T}^{3}$ case) there are no constraints left so that the system can be completely described by the time dependent Hamiltonians that we have found. This is in contrast with the situation for the 3-torus where in addition to the dynamics generated by the time dependent Hamiltonian there is an additional constraint in the system that must be appropriately taken into account.

A somewhat surprising fact is the possibility to describe both the $\mathbb{S}^{1} \times \mathbb{S}^{2}$ and $\mathbb{S}^{3}$ models by using smooth fields on the sphere $\mathbb{S}^{2}$. An interesting possibility that may teach us something in the case of $\mathbb{S}^{3}$ is to use a Hopf fibration to perform the Geroch reduction to get a $2+1$ dimensional description. This may be the subject of future work.

The dynamics of the global modes for the different spatial topologies is easy to obtain but there are significant differences depending on the topologies. Whereas in the $\mathbb{T}^{3}$ case the value of $\tilde{Q}$ and $\tilde{P}$ are just constants in the other cases $\tilde{P}$ is constant but $\tilde{Q}$ is a function of time.

We have been able to understand in very simple terms the appearance of both initial and final singularities in the spacetime metrics that solve the Einstein-Klein Gordon equations for these models from the point of view of the phase space description of the dynamics, in particular after the deparameterization process that we have followed. As we have seen there are natural variables with very simple gauge transformations ("time dynamics") that suggest canonical transformations that lead to the time dependent Hamiltonians describing the dynamics and explicitly show how the singularities appear. In the $\mathbb{S}^{1} \times \mathbb{S}^{2}$ and $\mathbb{S}^{3}$ topologies the function $\sin t$ in a denominator of the time-dependent Hamiltonian shows that both final and initial singularities are present whereas the $t$ denominator in the Hamiltonian for the 3-torus shows that only an initial (or final) singularity appears in this case.

\section{Acknowledgments}

The authors want to thank I. Garay and J. M. Martín García for discussions. Daniel Gómez Vergel acknowledges the support of the Spanish Research Council (CSIC) through a I3P research assistantship. This work is also supported by the Spanish MEC under the research grant FIS2005-05736-C03-02.

[1] R. H. Gowdy, Phys. Rev. Lett. 27, 826 (1971).

[2] R. H. Gowdy, Ann. Phys. 83, 203 (1974).

[3] C. W. Misner, Phys. Rev. D8, 3271 (1973).

[4] B. K. Berger, Ann. Phys. 83, 458 (1974). 
[5] B. K. Berger, Phys. Rev. D11, 2770 (1975).

[6] J. Cortez and G. A. Mena Marugán, Phys. Rev. D72, 064020 (2005).

[7] A. Corichi, J. Cortez, and G. A. Mena Marugán, Phys. Rev. D73, 084020 (2006).

[8] A. Corichi, J. Cortez, G. A. Mena Marugán, and J. M. Velhinho, Class. Quant. Grav. 23, 6301 (2006).

[9] G. A. Mena Marugán, Phys. Rev. D56, 908 (1997).

[10] C. G. Torre, Phys. Rev. D66, 084017 (2002).

[11] C. G. Torre, Class. Quant. Grav. 24, 1 (2007).

[12] J. D. Romano and C. G. Torre, Phys. Rev. D53, 5634 (1996).

[13] A. Corichi, J. Cortez, and H. Quevedo, Int. J. Mod. Phys. D11, 1451 (2002).

[14] M. Pierri, Int. J. Mod. Phys. D11, 135 (2002).

[15] J. F. Barbero G., D. G. Vergel, and E. J. S. Villaseñor, Phys. Rev. D74, 024003 (2006).

[16] J. F. Barbero G., I. Garay, and E. J. S. Villaseñor, Phys. Rev. Lett. 95, 051301 (2005).

[17] J. F. Barbero G., I. Garay, and E. J. S. Villaseñor, Phys. Rev. D74, 044004 (2006).

[18] A. Ashtekar and M. Pierri, J. Math. Phys. 37, 6250 (1996).

[19] C. Beetle, Adv. Theor. Math. Phys. 2, 471 (1998).

[20] J. L. Hanquin and J. Demaret, J. Phys. A: Math. Gen. 16, L5 (1983).

[21] M. Varadarajan, Phys.Rev. D75, 044018 (2007).

[22] P. S. Mostert, Ann. of Math. 65, 447 (1957); 66, 589 (1957).

[23] P. Chrusciel, Ann. Phys. 202, 100 (1990).

[24] R. Penrose and W. Rindler, Spinors and Space-Time, Cambridge University Press (1987).

[25] R. M. Wald, General Relativity, The University of Chicago Press, USA (1984).

[26] R. Geroch, J. Math. Phys. 12, 918 (1971).

[27] O. Rinne and J. M. Stewart, Class. Quant. Grav. 22, 1143 (2005).

[28] M. de León and P. R. Rodrigues, Methods of Differential Geometry in Analytical Mechanics, North Holland, Mathematics Studies 158 (1989).

[29] M. de León, J. C. Marrero, and D. M. de Diego, J. Phys. A: Math. Gen. 29, 6843 (1996).

[30] J. F. Barbero G., D. G. Vergel, and E. J. S. Villaseñor. In preparation. 